ARTICLE

DOI: $10.1038 / s 41467-017-01679-x$

\title{
Structural and spatial chromatin features at developmental gene loci in human pluripotent stem cells
}

Hiroki lkeda', Masamitsu Sone ${ }^{1,2}$, Shinya Yamanaka ${ }^{1,3} \&$ Takuya Yamamoto (D) 1,2,4

Higher-order chromatin organization controls transcriptional programs that govern cell properties and functions. In order for pluripotent stem cells (PSCs) to appropriately respond to differentiation signals, developmental gene loci should be structurally and spatially regulated to be readily available for immediate transcription, even though these genes are hardly expressed in PSCs. Here, we show that both chromatin interaction profiles and nuclear positions at developmental gene loci differ between human somatic cells and hPSCs, and that changes in the chromatin interactions are closely related to the nuclear repositioning. Moreover, we also demonstrate that developmental gene loci, which have bivalent histone modifications, tend to colocalize in PSCs. Furthermore, this colocalization requires PRC1, PRC2, and TrxG complexes, which are essential regulatory factors for the maintenance of transcriptionally poised developmental genes. Our results indicate that higher-order chromatin regulation may be an integral part of the differentiation capacity that defines pluripotency.

\footnotetext{
${ }^{1}$ Department of Life Science Frontiers, Center for iPS Cell Research and Application (CiRA), Kyoto University, Sakyo-ku, Kyoto 606-8507, Japan. ${ }^{2}$ Institute for Integrated Cell-Material Sciences (WPI-iCeMS), Kyoto University, Sakyo-ku, Kyoto 606-8507, Japan. ${ }^{3}$ Gladstone Institute of Cardiovascular Disease, San Francisco, CA 94158, USA. ${ }^{4}$ AMED-CREST, AMED 1-7-1 Otemach, Chiyodaku, Tokyo 100-0004, Japan. Correspondence and requests for materials should be addressed to T.Y. (email: takuya@cira.kyoto-u.ac.jp)
} 
O ne prominent aspect of stem cells is their ability to differentiate into other cell types. Specifically, pluripotent stem cells (PSCs), including embryonic stem cells (ESCs) and induced pluripotent stem cells (iPSCs), can give rise to almost all cell types within an animal's body. In the pluripotent state, developmental genes are rarely expressed in PSCs, but should be properly transcribed in response to extracellular differentiation cues. Therefore, in order to understand the differentiation ability of PSCs, it is important to know how developmental genes are regulated in order to promptly undergo transcription upon stimulation.

Epigenetic regulation by histone modification plays critical roles in transcriptional programs that govern various biological processes. In PSCs, distinct histone modification regions, termed as bivalent domains, have been observed in the promoters of many developmental genes ${ }^{1-5}$. Bivalent domains have both transcriptionally active ( $\mathrm{H} 3 \mathrm{~K} 4 \mathrm{me} 3)$ and repressive $(\mathrm{H} 3 \mathrm{~K} 27 \mathrm{me} 3)$ histone marks, which are independently catalyzed by the trithorax group (TrxG) and polycomb repressive complex 2 (PRC2) complexes, respectively ${ }^{6-8}$. Moreover, polycomb repressive complex 1 (PRC1), which has ubiquitin ligase activity, binds to bivalent domains by recognizing $\mathrm{H} 3 \mathrm{~K} 27 \mathrm{me} 3$ and maintains the inactivation state of developmental genes ${ }^{9}$. Notably, bivalent domains are frequently occupied by paused RNA polymerase $\mathrm{II}^{10}$, 11 , suggesting that bivalency is a mark of developmental genes that are in transcriptionally silent but poised states in PSCs. Most of the bivalent gene loci in PSCs lose either active (H3K4me3) or repressive (H3K27me3) marks upon PSC differentiation ${ }^{1}$. Conversely, during somatic cell reprogramming, bivalency at developmental gene loci is reestablished in their promoters ${ }^{12}$. Furthermore, knockout experiments have implied that epigenetic modifiers that establish bivalency might be required for developmental plasticity ${ }^{13-15}$. Thus, the regulation of bivalent modification is closely related to the cellular differentiation of PSCs.

In addition to histone modifications, higher-order chromatin arrangements through three-dimensional (3D) architecture and subnuclear localization are also key factors for the control of transcription. Previous studies have shown that upon the induction of PSCs, pluripotency gene loci, including Nanog, are repositioned from the nuclear periphery to the nuclear interior ${ }^{16}$, and that the promoter-enhancer looping structure of the pluripotency genes is reorganized before transcriptional activation ${ }^{17}$, suggesting a crucial role of chromatin interaction during the induction. However, it remains unclear whether higher-order chromatin structures at developmental gene loci are altered during somatic cell reprogramming and how these structures are regulated in PSCs.

Generally, chromosome conformation capture (3C)-based methods are widely used to determine the physical interactions of distant DNA loci ${ }^{18}$. These methods quantify chimeric DNA fragments that are derived from proximity ligation events. Among them, circular chromosome conformation capture sequencing (4C or $4 \mathrm{C}$-seq) can identify spatial interactions of a single genomic locus on a genome-wide scale ${ }^{19-21}$. Since $4 \mathrm{C}$-seq focuses on one genomic locus as the analysis target, the required total read number for analyzing chromatin interactions with $4 \mathrm{C}$ seq is much smaller than that with the $\mathrm{Hi}-\mathrm{C}$ method, which detects all combinations of genomic interactions ${ }^{22}$. However, conventional $4 \mathrm{C}$-seq has some disadvantages in the library preparation, which requires inverse PCR using a circularized $3 \mathrm{C}$ library as a template, prior to quantification by massively parallel sequencing. This inverse PCR results in the amplification of DNA fragments that have the same $5^{\prime}$ and $3^{\prime}$ sequences derived from the primers, and the artificial PCR duplicates prevent quantitative analysis of the $4 \mathrm{C}$-seq data. Furthermore, the circular 3C library construction process contains DNA that has been digested with a restriction enzyme (typically, a 4-bp recognition enzyme); therefore, it has a potential bias associated with the uneven distribution of the enzyme recognition sequence. In addition, conventional $4 \mathrm{C}$-seq is restricted to one genomic locus and cannot reveal the interaction profiles at multiple regions in parallel.

In this study, to investigate the global chromatin interaction profiles around dozens of developmental gene loci in detail, we develop a multiplexed Splinkerette ${ }^{23}$ chromosome conformation capture combined with high-throughput sequencing (ms4C-seq) by improving upon previously reported $4 \mathrm{C}$ methods ${ }^{17,24,25}$. The ms4C-seq allows simultaneous analysis of chromatin interactions from multiple viewpoints while limiting the effects of PCR and sequence bias. Using ms4C-seq, we examine the genome-wide chromatin interaction profiles of multiple developmental gene loci before and after human somatic cellular reprogramming. Our results demonstrate that the global interaction profiles of developmental genes dynamically change during the reprogramming process. Furthermore, we find that the position of pluripotency gene loci and also developmental gene loci change from the nuclear periphery in somatic cells to the nuclear interior in human PSCs (hPSCs), and these changes are accompanied by changes in the nuclear positions of their interaction target loci. Finally, we show that bivalent promoters have a tendency to colocalize with one another in hPSCs, and that the knockdown of components of PRC1, PRC2, or TrxG complexes disrupts bivalent gene colocalization, thereby suggesting that the histone modification machinery plays an instructive role in higher-order chromatin organization. Thus, our findings illuminate the regulatory mechanisms of developmental genes that are crucial for the cellular differentiation of hPSCs.

\section{Results}

ms4C-seq reveals chromatin structures at bivalent gene loci. It has been shown that pluripotency gene loci are dynamically reorganized before their reactivation during somatic cell reprogramming ${ }^{17,26}$. Here, we focused our attention on developmental genes, which are frequently marked in PSCs by both active ( $\mathrm{H} 3 \mathrm{~K} 4 \mathrm{me} 3)$ and repressive $(\mathrm{H} 3 \mathrm{~K} 27 \mathrm{me} 3)$ histone modifications, called bivalent domains ${ }^{1-5}$. To examine the chromatin interaction profiles of the developmental genes (especially bivalent genes) at high resolution, we tried to perform a 4C- (circularized chromosome conformation capture) based massively paralleled sequenced method (4C-seq). Many versions of $4 \mathrm{C}$-seq have been reported to overcome the disadvantages of $4 \mathrm{C}$-seq described above by introducing several modifications, such as sonication-

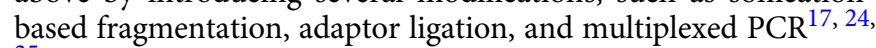
25 . Thus, we combined the beneficial features of those modified methods and developed a version of $4 \mathrm{C}$-seq, termed Multiplexed Splinkerette ${ }^{23}$ chromosome conformation capture combined with high-throughput sequencing (ms4C-seq) (Fig. 1a; see Methods). To avoid nonspecific priming in the enrichment of bait loci by adaptor ligation-mediated PCR, we employed splinkerette-PCR ${ }^{23}$.

To select the baits for our analysis with ms4C-seq systematically, we used publicly available data sets for chromatin-state segmentation determined by $\mathrm{ChromHmm}{ }^{27}$, which integrates multiple genome-wide data sets such as ChIP-seq data across multiple cell types using a multivariate Hidden Markov Model (HMM) to characterize chromatin states. In a previous report that applied ChromHMM to nine ChIP-seq plus input ENCODE data across nine cell types ${ }^{28}$, all human genomic regions (200 base pair interval) were assigned to 15 chromatin states including a "Poised Promoter" state. Comparison with ChIP-seq data sets ${ }^{29}$ showed that virtually all the genes whose promoter regions $( \pm 250$ bp from transcription start sites (TSS)) are categorized into a "Poised_Promoter" state by ChromHmm have both H3K4me3 
and $\mathrm{H} 3 \mathrm{~K} 27 \mathrm{me} 3$ modifications in their promoters. Therefore, hereafter, we designate these genes as "bivalent genes" in PSCs and human fibroblasts (HFs) (Fig. $1 \mathrm{~b}$ for PSCs and
Supplementary Fig. 1 for HFs). Likewise, we defined genes that have promoters categorized into "Active/Weak Promoter" and "Repressed/Heterochrom" by ChromHmm as "active genes" and

a

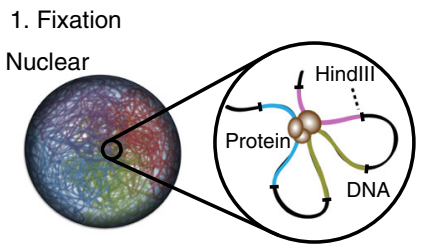

4. Sonication

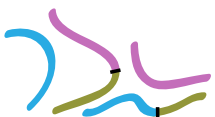

Splinkerette
adaptor

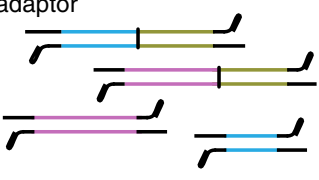

3. Ligation
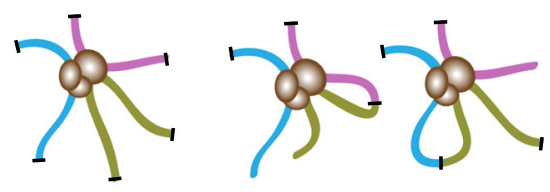

6. PCR amplification

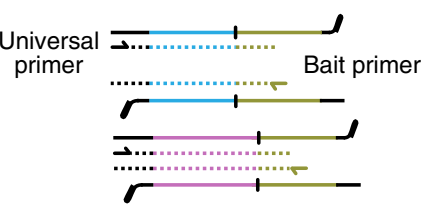

7. Nested PCR

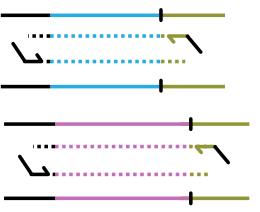

b

PSC

8. Deep sequencing and data analysis

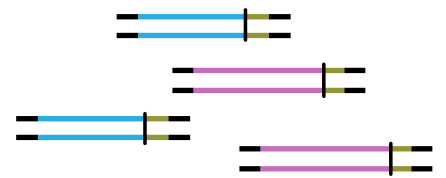

$\%$ Occupancy of chromatin states at promoter regions ( $\pm 250 \mathrm{bp}$ from TSS)

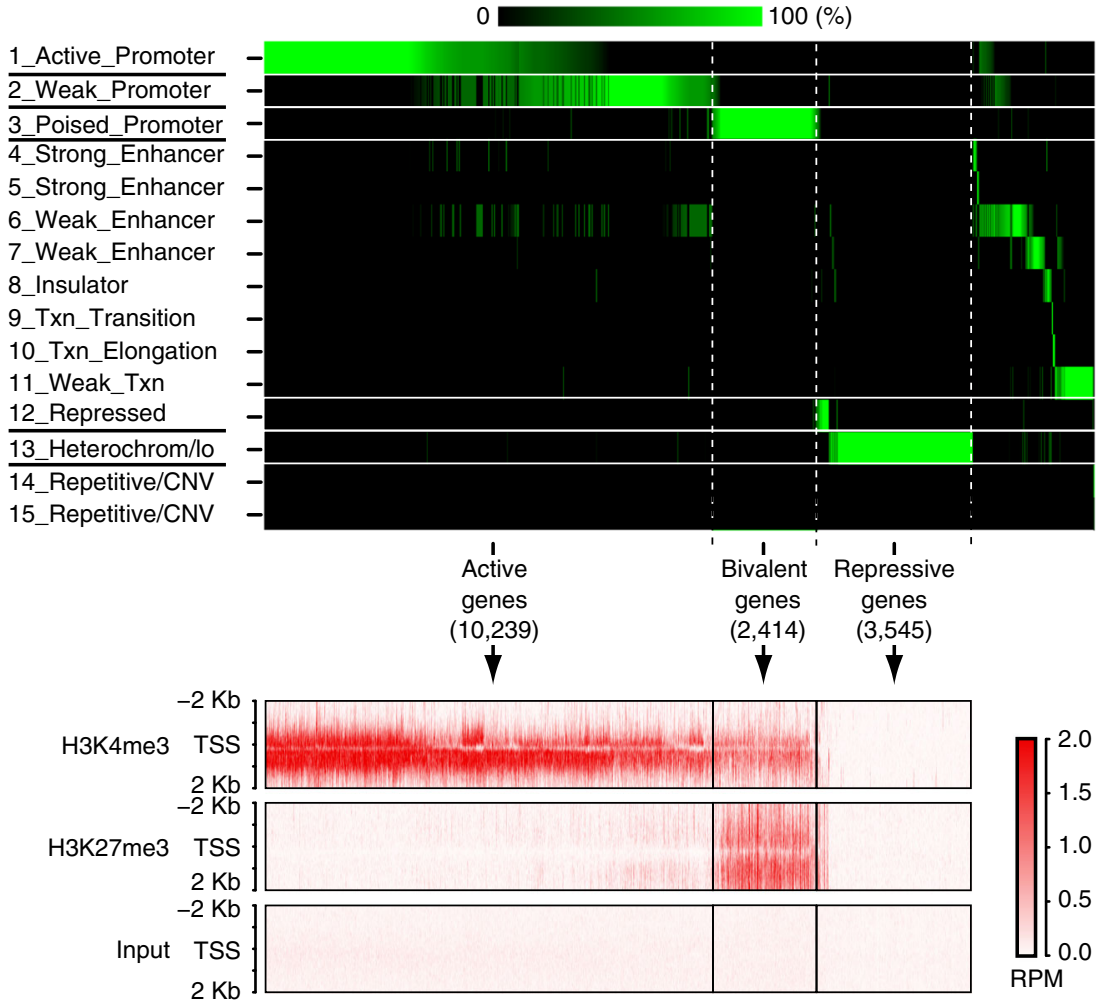

Fig. $1 \mathrm{~ms} 4 \mathrm{C}$-seq and definition of genes by chromatin states. a Schematic diagram of ms4C-seq. Step 1: fixation of a nucleus with formaldehyde. Step 2: digestion of DNA with Hindlll restriction enzyme. Step 3: preparation of chimeric fragments that are derived from physical proximity ligation of DNA. Step 4: fragmentation of those chimeric fragments. Step 5-7: ligations of Splinkerette adapter ${ }^{22}$ and amplifications of target loci by nested PCR with a universal primer for the adaptor and specific primers designed for bait loci. Step 8: deep sequencing by a next-generation sequencer. See also Supplementary Table 1 and Methods for details. b Definition of transcriptional states of 18,984 genes (bivalent, active, repressive, and other gene groups). The top heat map represents the occupancy of each chromatin state (\%) within TSS \pm 250 bp for all refseq genes in PSCs (ESCs). Bottom heatmaps indicate H3K4me3 and H3K27me3 modification profiles (RPM values) around TSS for active, repressive, and bivalent genes defined by chromatin states in PSCs (ESCs). Histone modification profiles are shown within TSS $\pm 2 \mathrm{~Kb}$ 
a

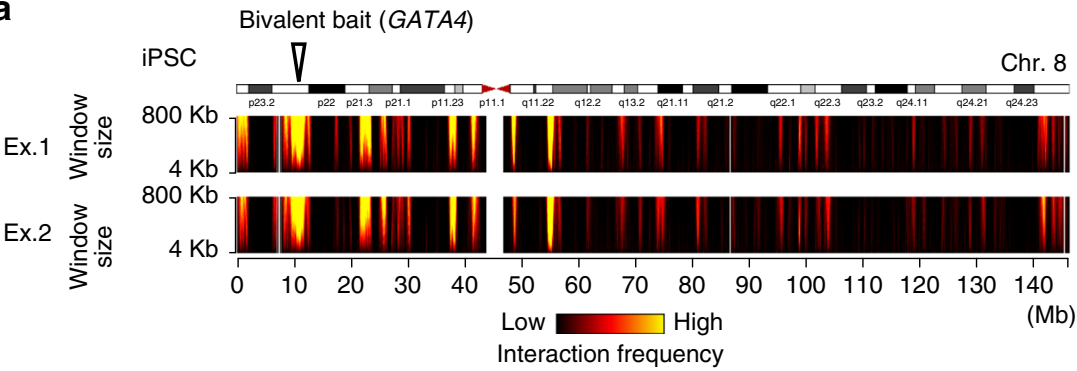

b

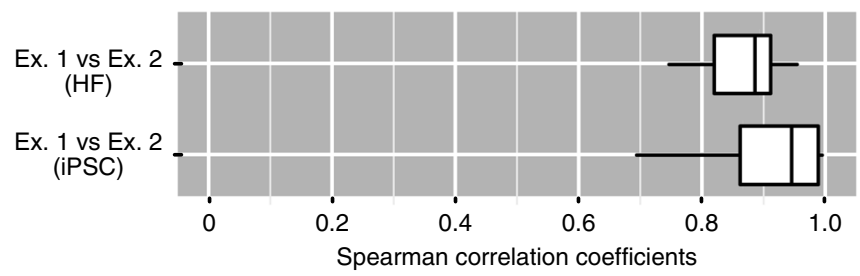

C
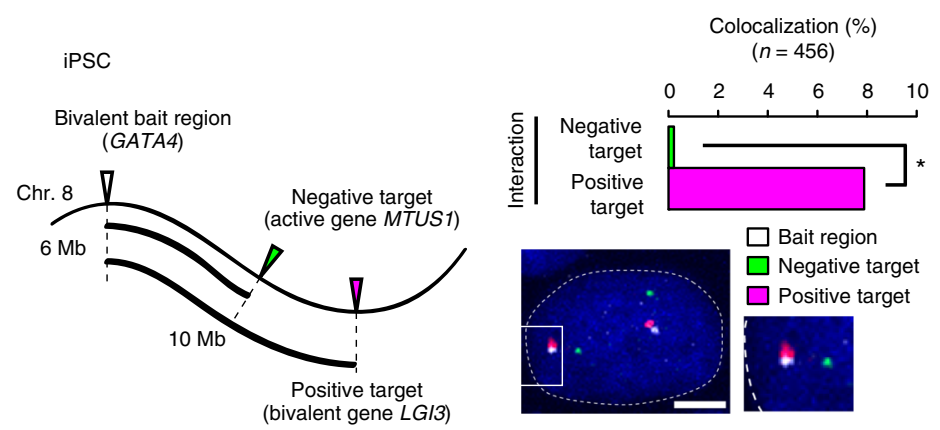

Fig. 2 Examination of chromatin interaction profiles at bivalent gene loci. a Cis-chromosomal interaction profiles at the GATA4 (bivalent in PSCs) gene locus in hiPSCs. Interaction frequencies of the GATA4 gene locus, as determined by ms $4 C$-seq, are presented by the domainogram in biological duplicates (Ex. 1 and Ex. 2). The color scale represents the $\log _{10}\left(p\right.$-value) interaction frequencies from low significance $(p=1)$ to high significance $\left(p=10^{-10}\right)$, with colors from black to yellow, respectively. $\mathbf{b}$ Analysis of the reproducibility of two independent ms4C-seq experiments in HFs (HDFs) and iPSCs. Boxplots indicate the distribution of the Spearman correlation coefficients of interaction signals among biological replicates (Ex. 1 vs. Ex. 2). c Analysis of chromatin interactions with 3D DNA FISH in iPSCs. The left panel illustrates the positions of positive (bivalent gene LGI3 in PSCs) and negative (active gene MTUS1 in PSCs) interaction target loci relative to the bait (bivalent gene GATA4 in PSCs) locus on the genome. The bar graph in the right panel shows the colocalization percentage between the GATA4 locus and the positive (magenta) or negative (green) interaction loci ( $n=456$, Fisher's exact test, ${ }^{\star} p<1 \times 10^{-8}$ ). The right lower panel shows a nuclear image of 3D DNA FISH with Z-projection. Nuclei are stained with Hoechst (blue). The bait locus (GATA4), interaction-positive locus (LGI3), and interaction-negative locus (MTUS1) are indicated in white, magenta, and green, respectively. Scale bar, $5 \mu \mathrm{m}$. See also Supplementary Fig. 2 and Supplementary Tables 2 and 3

"repressive genes", respectively, in both PSCs and HFs (Fig. 1b for PSCs and Supplementary Fig. 1 for HFs). Consistent with a previous report ${ }^{1,30,31}$, the number of bivalent genes in PSCs is much greater than that in somatic cells (HFs) because the gene number of the bivalency lost is greater than that of the bivalency reformation during PSC differentiation.

To investigate the chromatin interaction feature in bivalent gene loci, as the bait regions of ms4C-seq, we selected 29 bivalent, 31 active, 17 repressive, and 12 other genes from the gene groups in PSCs that we defined above (Fig. 1b; Supplementary Tables 1, 2). The 29 bivalent genes in PSCs include typical developmental regulators for ectoderm, mesoderm, and endoderm ${ }^{32-35}$. We performed ms4C-seq for all 89 bait loci on human iPSCs (hiPSCs; generated with episomal vectors), and for 42 bait loci out of 89 bait loci on their original HFs. Using the data from each bait that met our strict criteria for quality control (Supplementary Tables 2, 3; see also Methods), we created domainograms to visualize the interaction profiles ${ }^{36,37}$ (Fig. 2a; Supplementary Fig. 2a). These profiles clearly demonstrated that ms4C-seq achieves high reproducibility between two independent biological replicates in
HFs and hiPSCs. Moreover, the Spearman correlation coefficients for the chromatin interaction frequencies between biological replicates also confirmed the high reproducibility of ms4C-seq (Fig. 2b; Supplementary Table 4). We then assessed the results of ms4C-seq by 3D DNA fluorescence in situ hybridization (FISH) and confirmed the physical interactions at the single-cell level in both HFs and hiPSCs (Fig. 2c; Supplementary Fig. 2b). Furthermore, 3C-qPCR assays independently confirmed our ms4C-seq data, showing that the HOXA13 locus frequently interacts with HOXA3 and HOXA5 in hiPSCs but not in HFs (Supplementary Fig. 2c). Taken together, our ms4C-seq data are highly reliable for analyzing the genome-wide interaction profiles of bivalent regions before and after cellular reprogramming.

Chromatin structures reorganize at bivalent gene loci. During mouse iPSC induction, enhancer-promoter looping formation at the Nanog locus is reestablished before the genes are expressed ${ }^{17}$, possibly indicating that chromatin remodeling causes changes in gene expression. In order to investigate the relationship between 
a
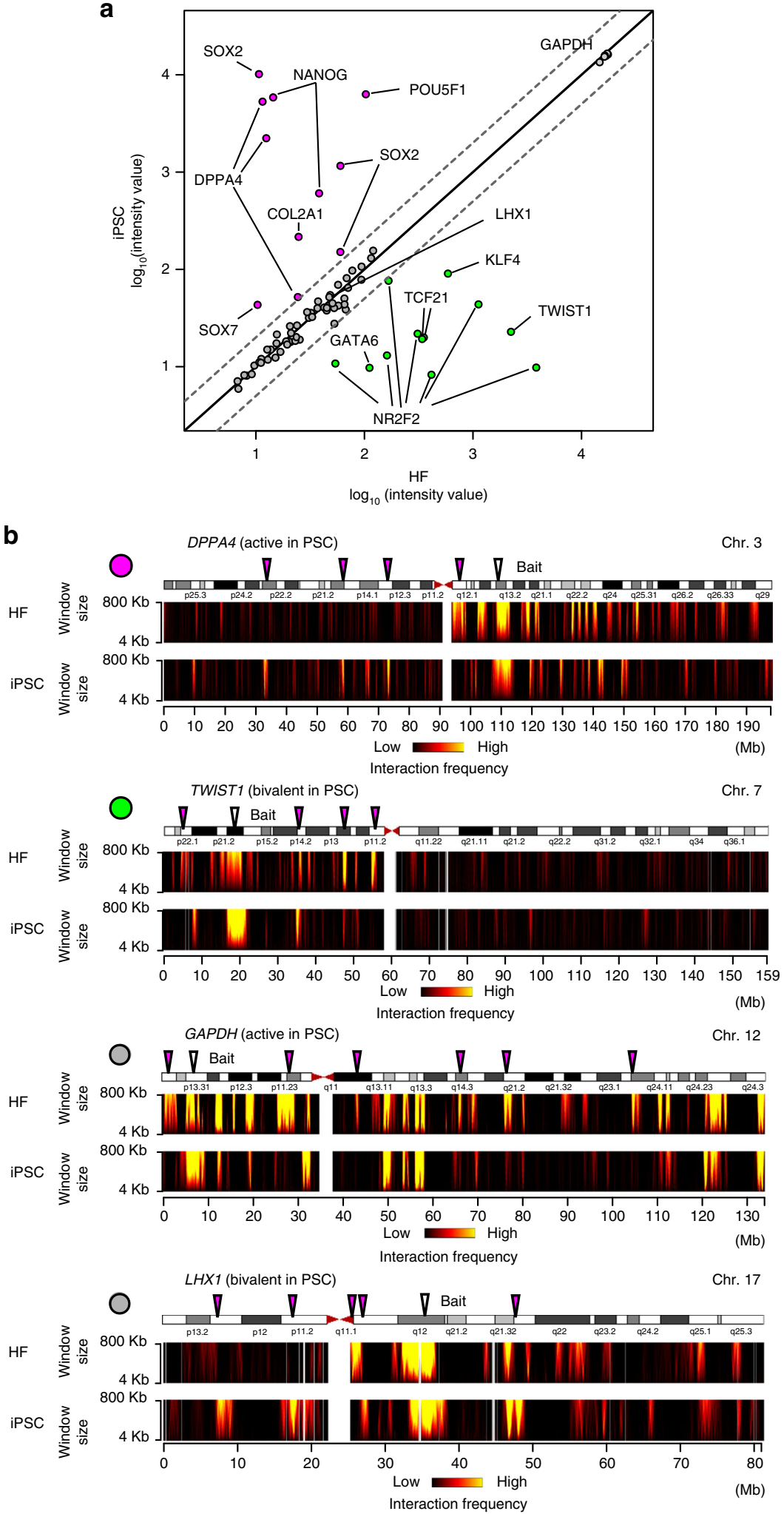

Fig. 3 Chromatin interaction profiles at bivalent gene loci in somatic cells and hPSCs. a Expression profiles of bait genes in iPSCs and their original HFs (HDFs). The scatter plot represents the $\log _{10}$ signal intensity of probe sets for Affymetrix GeneChip Array (HG-U133_Plus_2). A single gene is sometimes represented by multiple probe sets corresponding to different isoforms and ESTs derived from the same gene locus. Thus, some genes have multiple dots (probe sets). Two dashed lines indicate the two-fold changes in gene expression levels between HFs (HDFs) and hiPSCs. The dots are color-coded as category 1 (magenta), category 2 (green), and category 3 (gray) on the basis of the fold change of the signal intensity. b Cis-chromosomal interaction profiles of the bait loci (DPPA4, TWIST1, GAPDH, and LHX1). The interaction frequencies of baits are shown by domainograms ${ }^{37}$. DPPA4 and GAPDH are active genes in PSCs. TWIST1 and LHX1 are bivalent genes in PSCs. The arrowheads indicate bait loci (white) and representative interaction regions that differ between HFs (HDFs) and hiPSCs (magenta). The left upper dots are colored according to the definition in a 
chromatin structure and gene expression, we compared changes in the interaction profiles and gene expression profiles before and after hiPSC induction. The bait genes as viewpoints were divided into three groups: genes with higher (category 1), lower (category 2 ), and similar (category 3) expression in hiPSCs than HFs (Fig. 3a). We found that the interaction profiles for genes in all three categories dynamically changed before and after reprogramming (Fig. 3b; Supplementary Fig. 3). These results indicate that the chromatin interaction profiles of various bait gene loci are remodeled during somatic cell reprogramming regardless of changes in the expression at the bait genes.

Reposition of bivalent gene loci by cellular reprogramming. Pluripotency-related gene loci change their nuclear positions between the periphery and interior during the differentiation of mouse $\mathrm{ESCs}^{38}$, and this repositioning is important for transcriptional control ${ }^{39}$. However, little is known about the nuclear chromatin repositioning where the bivalency was restored in the reprogramming process. To investigate the subnuclear localization of bivalent gene loci in HFs and PSCs, we examined the distribution of lamina-association signals in active, repressive, and bivalent gene loci in HFs and PSCs using publically available data sets of LaminB1 DamID signals ${ }^{40,41}$ as a surrogate for peripheral localization, which was confirmed by DNA FISH experiments $^{38}$. Consistent with previous studies that reported the nuclear localizations of gene loci are closely related to transcription (active genes in the nuclear interior and inactive genes in the nuclear periphery) $39,42,43$, we observed that active and repressive genes were biased to the nuclear interior and periphery, respectively, in both HFs and PSCs (Fig. 4a). Notably, while bivalent genes in HFs tended to localize to the nuclear periphery, bivalent genes in PSCs were biased to the nuclear interior, suggesting that the regulatory mode of the nuclear localization for bivalent genes in HFs differs from those in PSCs. Next, we examined the relationship between the changes of chromatin states and those of nuclear localization and found that when the gene states are changed from repressive in HFs to active in PSCs, the distribution of the lamina-association signals becomes biased to the nuclear interior in PSCs (Fig. 4b, magenta lines). Moreover, the gene loci whose states changed from repressive in HFs to bivalent in PSCs behaved similarly to those changed to active in PSCs (Fig. 4b, black and magenta lines), even though bivalent genes were not transcriptionally activated in PSCs (Fig. 4c). These results suggested that repressed genes in HF changed their position from the nuclear periphery toward the interior in hPSCs not only when they became active but also when they became bivalent. Our data also showed that when bivalent loci as baits changed their position from the nuclear periphery of HFs to the interior of hPSCs, the interaction target regions of the baits likewise changed (Fig. 4d, e; Supplementary Fig. 3). These results imply that the reestablishment of $3 \mathrm{D}$ chromatin structures is accompanied by the reorganization of chromatin states and chromatin repositioning in the nucleus before and after cellular reprogramming.

Bivalent gene loci colocalize in pluripotent stem cells. Transcriptionally active gene loci are known to colocalize ${ }^{44-46}$ with one another. Because many active genes are marked by H3K4me3 in their promoter regions 3,47 , genomic regions with histone modification $\mathrm{H} 3 \mathrm{~K} 4 \mathrm{me} 3$ may be expected to colocalize. In fact, our $\mathrm{ms} 4 \mathrm{C}$-seq data also showed that pluripotency-related genes such as NANOG, DPPA4, and SOX2 (active genes in PSCs) interact with genomic regions with the H3K4me3 modification (Supplementary Fig. 4). To investigate the histone modification profiles around interaction targets of bivalent bait genes in PSCs, we compared our ms4C-seq data with a genome-wide data set of various histone modifications in hPSCs. Notably, we found that several interaction regions of bivalent bait loci often overlapped with the promoter regions of other bivalent genes (Fig. 5a; Supplementary Fig. 5). For example, in hiPSCs, the PAX6 bait region interacted with bivalent domains, including the promoters of WT1 and ALX4 (Fig. 5a). These observations indicate that bivalent genes localized together in hPSCs. To further support this notion, we calculated the enrichment of bivalent genes in the interaction regions from the perspective of the bivalent bait loci. Our data demonstrated that bivalent genes are enriched in the interaction targets of bivalent genes, but not in those of active or repressed genes (Fig. 5b, c). Importantly, while significant enrichments of active and repressive genes were observed in the interaction targets of active and repressive gene loci (baits), respectively (Supplementary Fig. 6a), no enrichment of active or repressive genes was observed in the interaction targets of bivalent gene loci (baits) (Supplementary Fig. 6a). These data suggested that bivalent genes preferentially interact with bivalent genes, but neither with active nor repressive genes. The colocalization of bivalent gene loci was also observed in single-cell analysis by $3 \mathrm{D}$ DNA FISH (Fig. 2c). To examine whether the colocalization of bivalent gene loci is true for other hPSC lines, we investigated the interaction frequencies of bivalent gene loci defined in PSCs by 3C-qPCR. In accordance with the results of the genome-wide analysis by ms4C-seq in hiPSCs, bivalent gene loci colocalized with one another in hPSCs in the nuclear interior (Fig. 5d, e). Together, our results strongly suggest that bivalent gene loci frequently colocalize in the nuclear interior of hPSCs.

Approximately $25 \%$ of bivalent genes are DNA methylation valley (DMV) genes ${ }^{48}$, which contain large hypomethylated regions. In addition to the above results, we observed that bivalent genes interacted with DMV genes (Supplementary Fig. 6b, c), suggesting that epigenetic regulation including histone modification and DNA methylation may contribute to the organization of higher-order chromatin structures in hPSCs.

Bivalent gene colocalization requires PcG and TrxG proteins. Histone modifiers, such as PRC1, PRC2, and TrxG, contribute to the maintenance of the poised state of bivalent gene loci in PSCs $^{6-8,11}$. To obtain mechanistic insights into the colocalization of bivalent gene loci, we focused on PRC1, PRC2, and TrxG protein complexes. We introduced small hairpin RNAs (shRNAs) that target RING1B, EED, and WDR5, which are components of the PRC1, PRC2, and TrxG complexes, respectively, into hiPSCs and performed ms4C-seq for eight selected bivalent bait loci to examine the effects of the shRNAs on the interaction profiles (Supplementary Fig. 7a). We confirmed that the expression of each protein targeted by the shRNAs was reduced by $50-90 \%$ (Supplementary Figs. 7b, 8). A large reduction in interaction frequency was not observed in any of the shRNA-treated samples at the resolution used in the domainogram analysis, indicating that the chromatin structures were largely maintained after the knockdown of EED, WDR5, or RING1B (Fig. 6a). In contrast, we noticed that normalized signals of ms $4 \mathrm{C}$-seq at several gene loci were significantly decreased after knockdown of the target genes (Fig. 6b). We examined the reduction rates of the interaction frequencies by shRNA knockdown experiments (shRNA/shNegative RPM) in the interaction target gene loci of bivalent bait gene loci and found that reduction rates associated with bivalent gene loci were lower than those associated with nonbivalent genes in independent sh1 and sh2 knockdown experiments for EED, WDR5, and RING1B with statistical significance (Fig. 6c). Then, we tried to identify the target genes whose interaction frequencies 
a
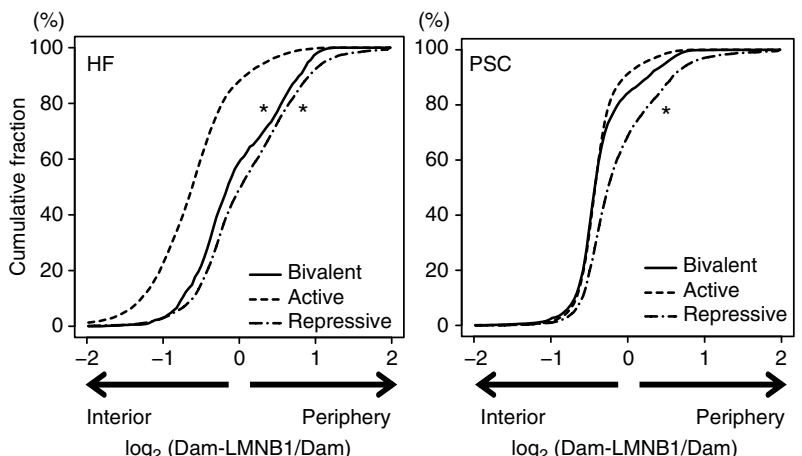

$\log _{2}$ (Dam-LMNB1/Dam)

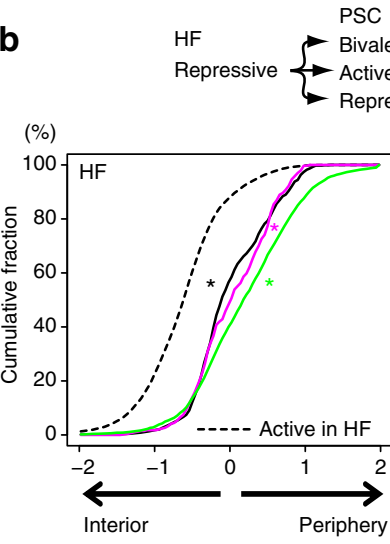

$\log _{2}$ (Dam-LMNB1/Dam)

PSC

Active
Repressive

(\%)

d

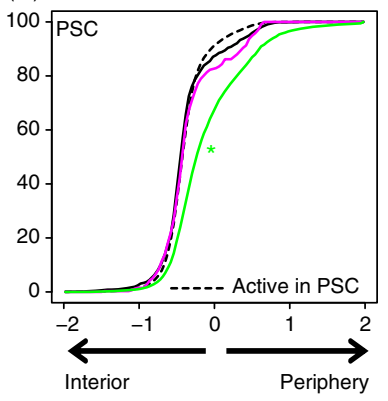

$\log _{2}$ (Dam-LMNB1/Dam)

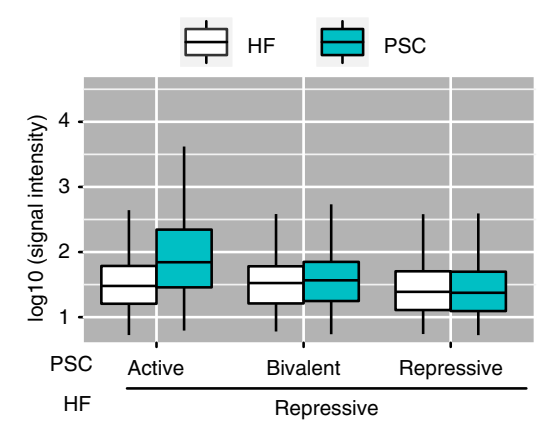

e

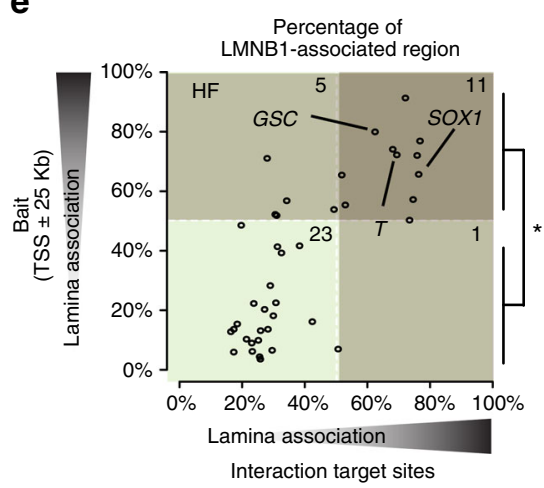

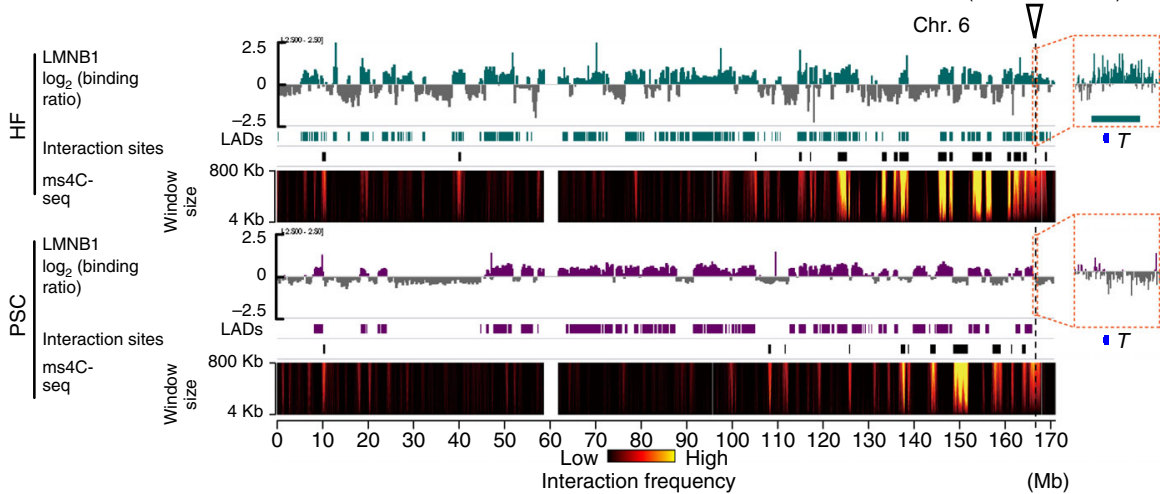

$(\mathrm{Mb})$

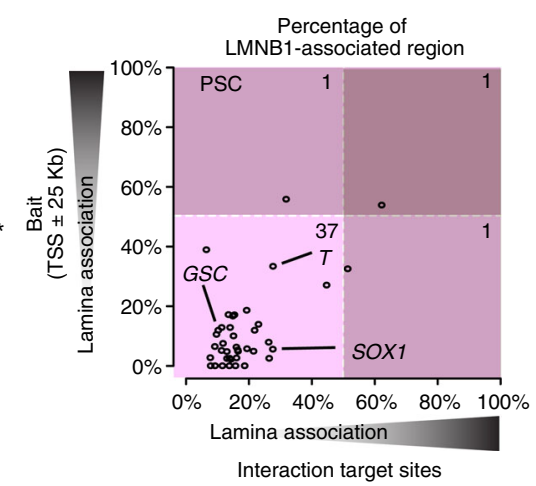

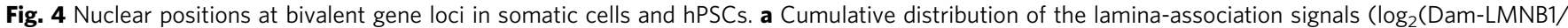
Dam) values ${ }^{39,40}$ ) at active (dashed line), repressive (dash-dot line), and bivalent (solid line) gene loci in $\mathrm{HFs}$ and PSCs (ESCs). ${ }^{\star} p<3.0 \times 10^{-16}$ for Mann-Whitney U-test using active genes for the comparisons. b Cumulative distribution of the lamina-association signals (log 2 (Dam-LMNB1/Dam) values 40,41 ) for the genes which change their states from repressive in HFs to active (magenta), repressive (green), or bivalent (black) in PSCs (ESCs). ${ }^{\star} p<3.0 \times 10^{-16}$ for Mann-Whitney $U$-test using active genes for the comparisons. comparison of expression values for the indicated gene groups in iPSCs and their original HFs (HDFs). The boxplots indicate the distribution of $\log _{10}$ (signal intensity) values. The box plots are colored by cell types. $\mathbf{d}$ The relationship between chromatin interaction profiles and lamina-association profiles at $T$ gene bait loci in HFs and hPSCs. The positive log 2 (Dam-LMNB1/ Dam) signals 40,41 are indicated by the green (HFs) or magenta (PSCs (ESCs)) bar chart above the HF and PSC tracks. Positive log ${ }_{2}$ (Dam-LMNB1/Dam) signals indicate lamina-associated regions. Rectangles below each signal track depict lamina-associated domains. The interaction frequencies determined by ms4C-seq in HFs (HDFs) and iPSCs are shown as described in Fig. 2a. e The subnuclear localization of the bait loci and their interaction target loci in HFs (top) and PSCs (iPSCs, bottom). Scatter plots present the strength of the lamina association of the bait gene loci (TSS $\pm 25 \mathrm{~kb}$ ) ( $y$-axis) and their chromatin interaction target sites ( $x$-axis) ( $U$-test, ${ }^{\star} p<3 \times 10^{-7}$ ). Bivalent baits indicating GSC, $T$, and SOX1 loci are shown as representative bait loci with interaction target loci located in the nuclear periphery in HFs and the nuclear interior in PSCs (ESCs)

were reduced by more than $30 \%$ in both independent knockdown experiments compared with negative control. Our results showed that many bivalent colocalization events were significantly disrupted by the knockdown of EED, WDR5, or RING1B compared to interactions with nonbivalent genes although the degree of the effects varied in each bait (Fig. 6d), and the knockdown effect was different depending on the bait (Supplementary Fig. 7c). These results led us to conclude that both $\mathrm{PcG}$ and TrxG proteins are important for the colocalization of bivalent gene loci, but that the effects are variable. 
a

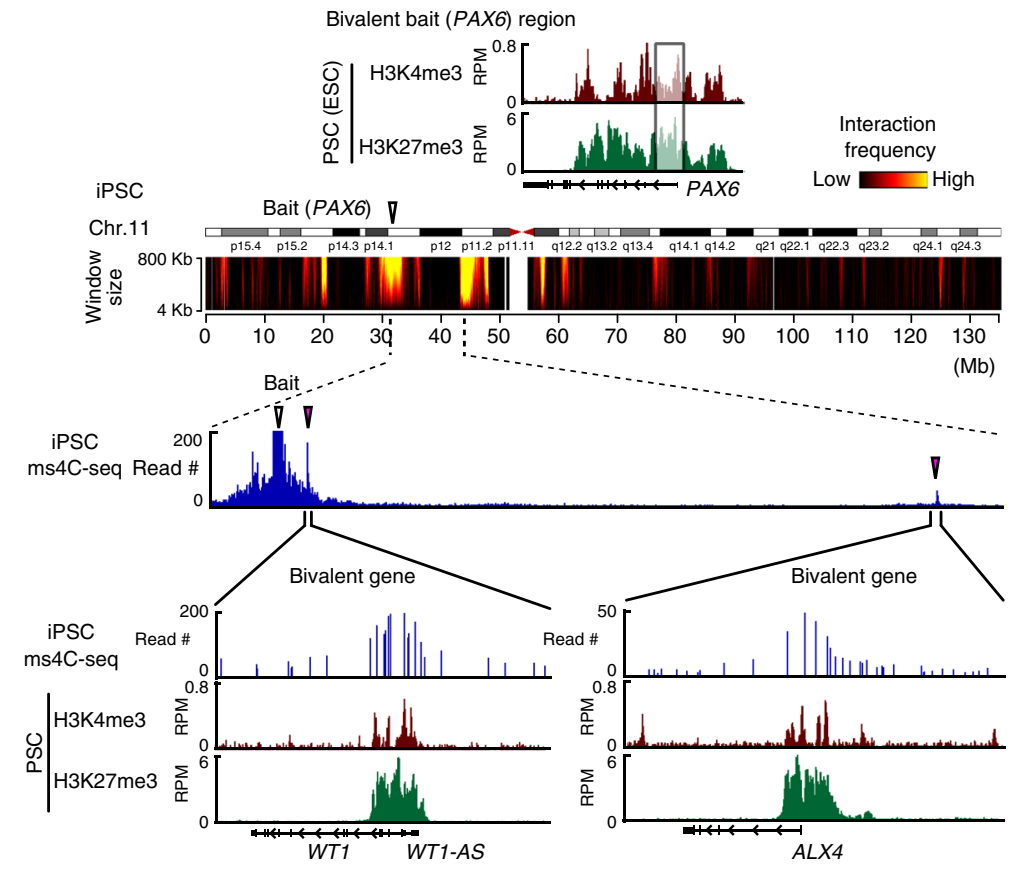

b
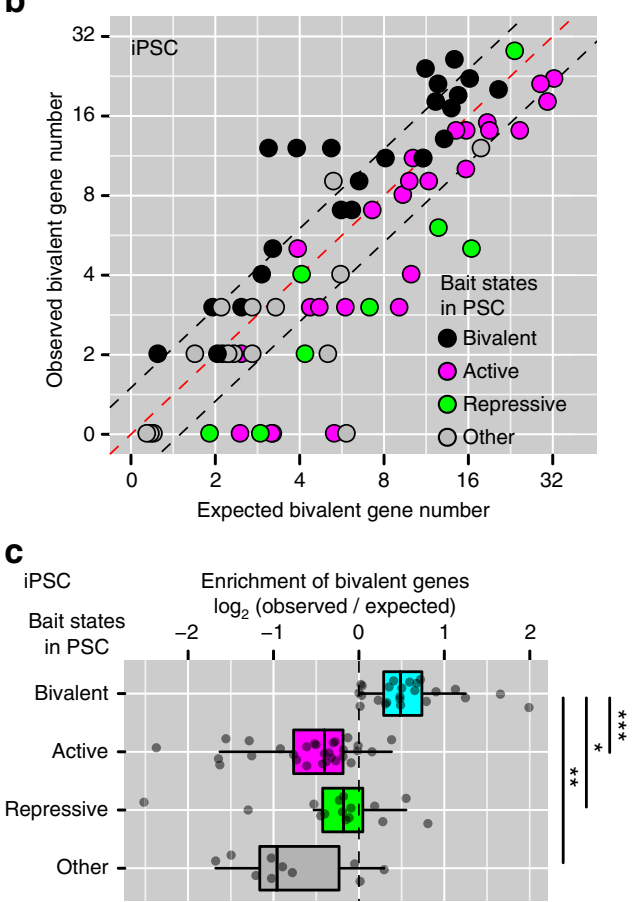

d
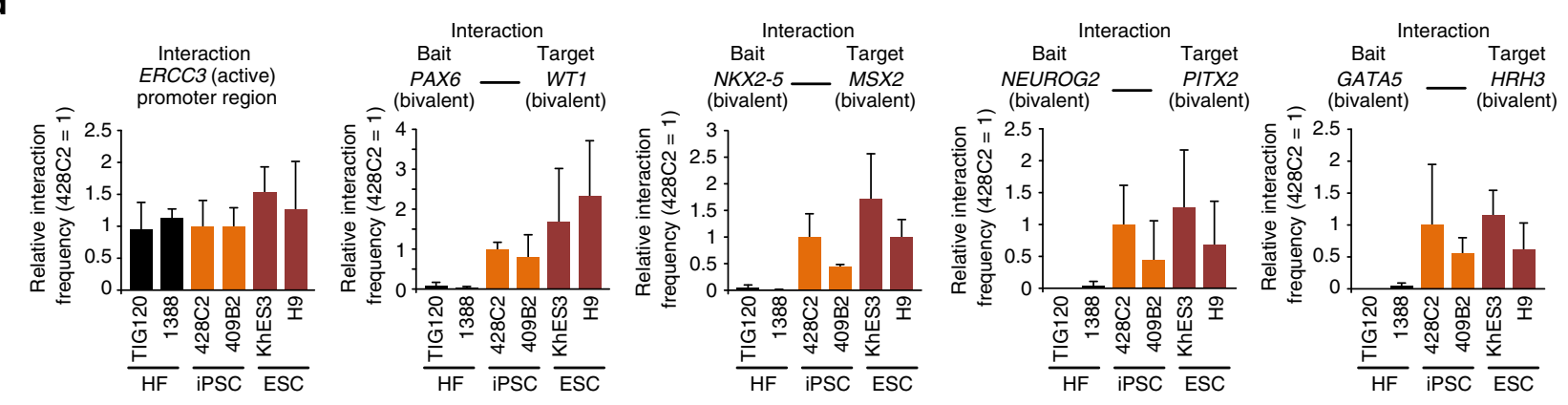

e

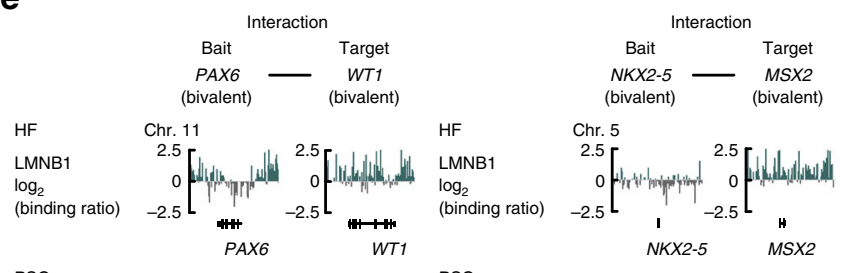

$\begin{array}{lrl}\text { PSC } & 2.5 \\ \text { LMNB1 } & 0 \\ \log _{2} & { }_{\text {(binding ratio) }} & -2.5\end{array}$
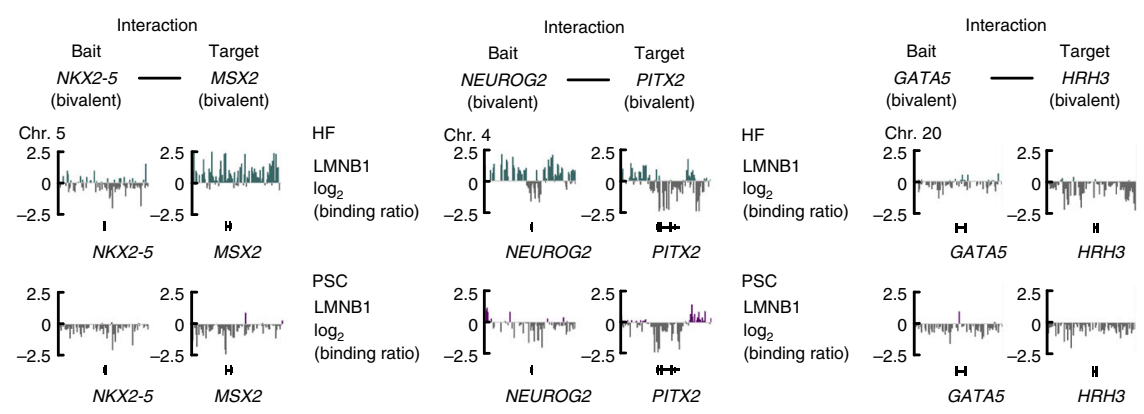

Fig. 5 Bivalent gene loci colocalize in hPSCs. a The distribution of histone modifications for H3K4me3 and H3K27me3 in the PAX6 bait locus and their target loci. ChIP-seq data are represented by RPM signals for H3K4me3 and H3K27me3 in ESCs below ms4C-seq interaction signals in iPSCs. b Bivalent gene numbers in the interaction target genes of active, repressive, and bivalent bait loci in iPSCs (428C2). The scatter plot shows the expected numbers of bivalent gene ( $x$-axis) and observed numbers of bivalent genes ( $y$-axis) in the interaction targets of each bait locus. The expected number was calculated by the proportion of bivalent genes present on the same chromosome as bait loci. Bait genes were divided into bivalent (black), active (magenta), repressive (green), and other (gray) in PSCs (ESCs) according to Fig. 1b. c The enrichment of bivalent genes in interaction target genes of bivalent bait loci in iPSCs (428C2). Box plots show $\log _{2}$ (observed frequency for bivalent genes)/(expected frequency for bivalent genes) in interaction targets of bivalent, active, repressive, and other bait gene loci (U-test, ${ }^{\star} p<3 \times 10^{-5},{ }^{\star \star} p<1 \times 10^{-5}$, and ${ }^{\star \star \star} p<1 \times 10^{-11}$ ). d Interaction frequencies of bivalent gene loci in $\mathrm{hESCs}$ (KhES3 and H9), hiPSCs (428C2 and 409B2), and their original HFs (TIG120 and 1388). Bar graphs indicate the interaction frequencies of the indicated gene loci determined by enhanced 3C-qPCR analysis. The interaction frequencies in the ERCC3 promoter region are shown as positive interaction controls. Data are shown as means \pm SD from three biological replicates. e Lamina-association profiles of colocalized bivalent gene loci are indicated by log ${ }_{2}$ (DamLMNB1/Dam) in HFs and PSCs (ESCs) ${ }^{40,41}$. See also Supplementary Figs. 4-6 


\section{Discussion}

In this study, we describe ms4C-seq using Splinkerette adaptor ${ }^{23}$, a 4C-based method that integrates the advantages of multiplexed PCR amplification ${ }^{21}$, adaptor-mediated PCR amplifications ${ }^{17}$, and sonication-based random DNA fragmentation ${ }^{24}$. ms4C-seq was able to reliably and simultaneously identify chromatin interactions at multiple bait loci in HFs and hiPSCs, while limiting PCR duplication bias (Fig. 2a, b; Supplementary Fig. 2a-c). a

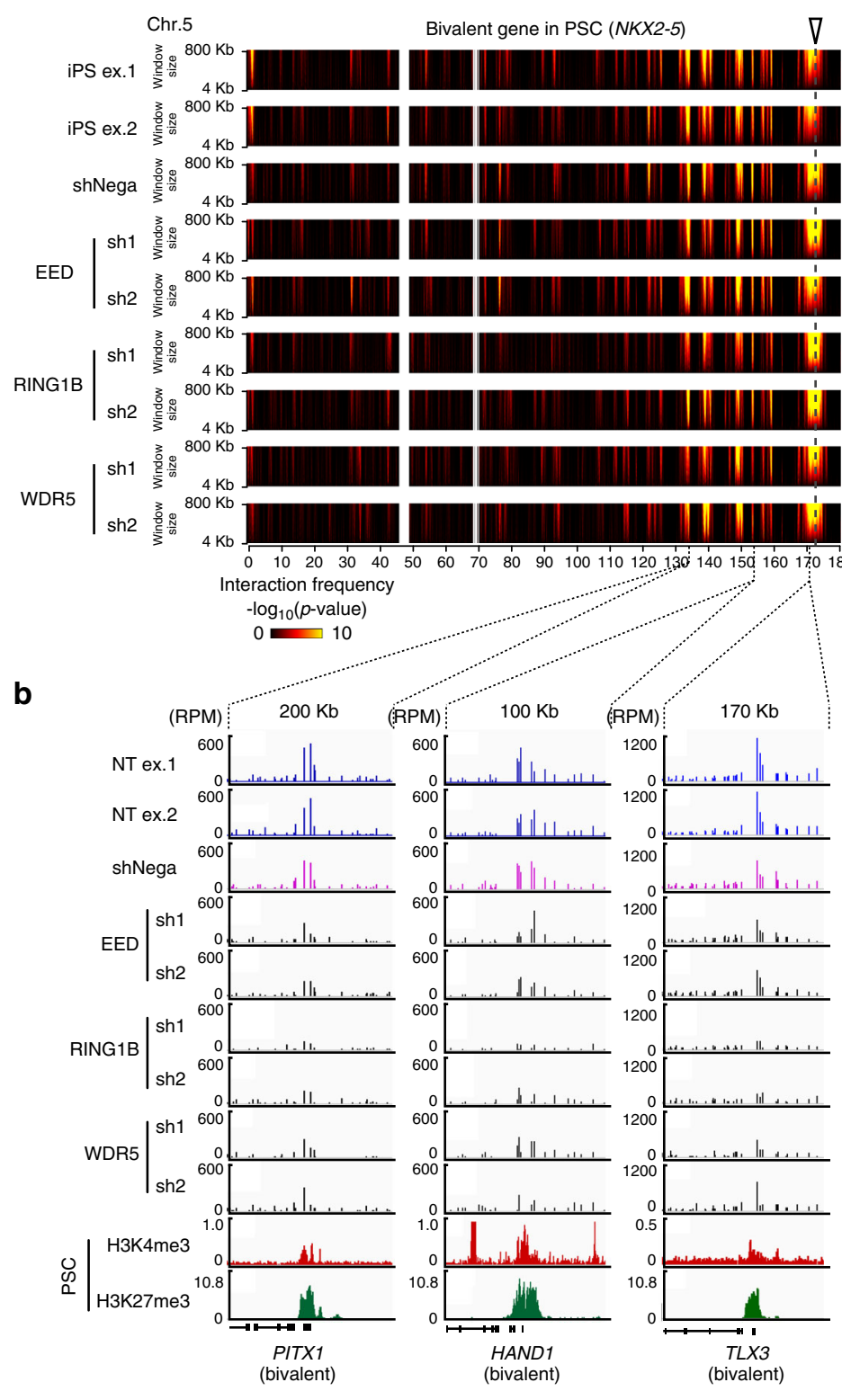

C iPSC Interaction $\quad$ Bivalent - Bivalent
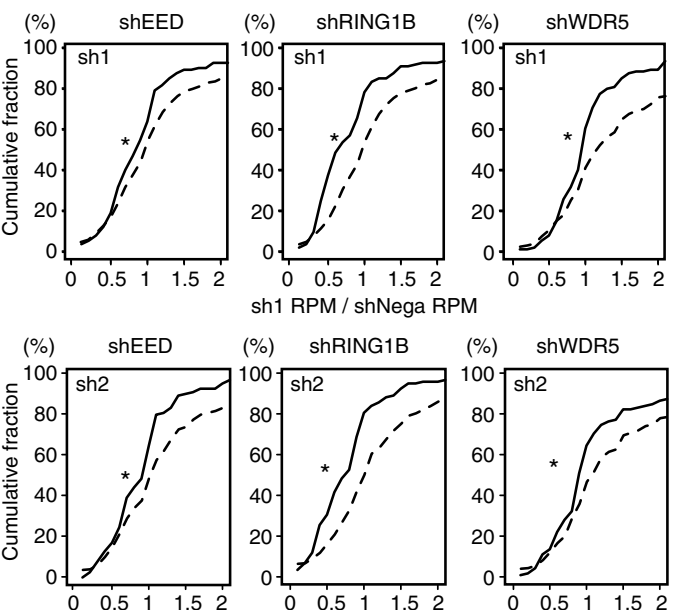

sh1 RPM / shNega RPM
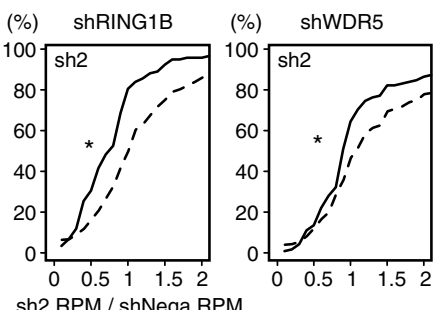

d

e
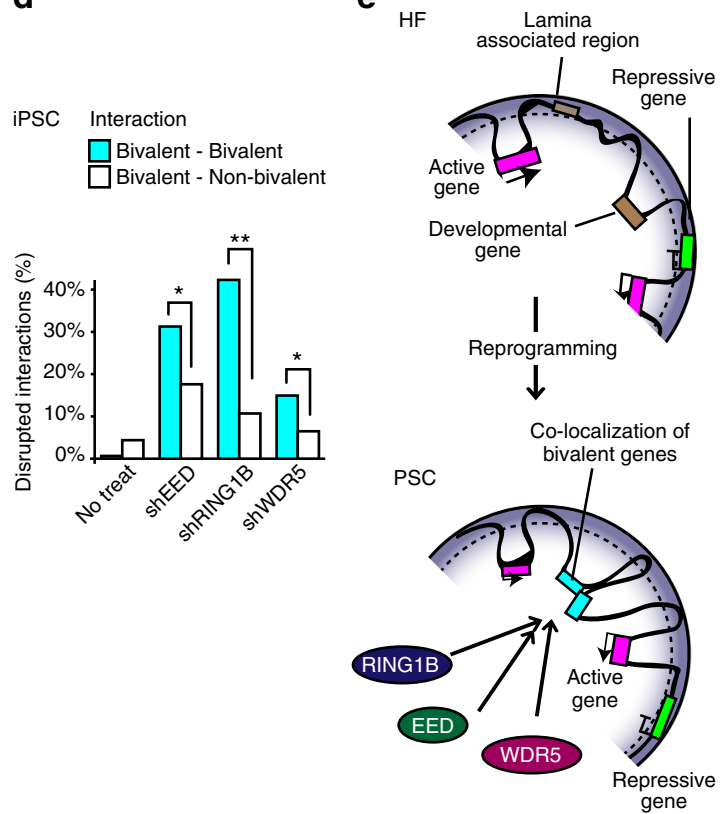

Fig. 6 EED, WDR4, and RING1B are important for the colocalization of bivalent gene loci. a Interaction profiles determined by ms4C-seq for the NKX2-5 gene locus in EED, WDR5, and RING1B KD iPSCs. Domainograms indicate cis-chromatin interaction frequencies at the NKX2-5 gene locus on an Mb scale in shRNA-treated iPSCs. $\mathbf{b}$ The disruption of chromatin interactions between bivalent gene loci by shRNA treatment. Bar plots indicate interaction signals (RPM) at interaction target gene loci of the NKX2-5 gene bait locus. ChIP-seq signals (RPM) for H3K4me3 and H3K27me3 histone modifications in PSCs (ESCs) are shown under the ms4C-seq interaction signals. Reduced interaction signals of the bait NKX2-5 gene locus are detected in bivalent gene loci (PITX1, HAND1, and TLX3) after shRNA treatment against EED, WDR5, or RING1B in PSCs (iPSCs). c Cumulative distribution of reduction rates for interaction signals (RPM values in TSS $\pm 25 \mathrm{~kb}$ ) in bivalent-bivalent (solid line) and bivalent-nonbivalent (dashed line) interactions by two independent knockdown (two different shRNAs) experiments for EED, RING1B, or WDR5 in PSCs (iPSCs). ${ }^{\star} p<0.03$ was assessed for bivalent-bivalent interactions vs. bivalent-nonbivalent interactions by two-sided Mann-Whitney U-test. d Disruption of bivalent gene colocalizations by the KD for EED, WDR5, or RING1B. Bar graphs show the percentages of colocalized bivalent genes (light blue) and nonbivalent genes (white) with interaction signals (RPM values in TSS \pm 25 $\mathrm{kb}$ ) that were reduced to at least $30 \%$ following both sh1 and sh2 treatments for EED, WDR5, and RING1B compared to shNegative treatment (Fisher's exact test, ${ }^{\star} p<0.01,{ }^{\star \star} p<1 \times 10^{-6}$ ). e A model for the changes in chromatin interactions and subnuclear localization around bivalent gene loci during somatic cell reprogramming. See also Supplementary Fig. 7 
Although the cis interactions identified in this study by $\mathrm{ms} 4 \mathrm{C}$-seq showed high reproducibility in biological duplicate experiments, the trans interactions did not (Supplementary Table 4). This poor reproducibility in trans interactions may have been due to spurious random ligation events among intermolecules in the insolution ligation step of the $\mathrm{ms} 4 \mathrm{C}$-seq preparation. Therefore, $\mathrm{ms} 4 \mathrm{C}$-seq may be improved by performing in-nucleus ligation in the 3 C library preparation ${ }^{49}$. We also observed a certain level of nonspecific amplification and unequal distribution of the number of sequenced reads among baits in the ms4C-seq data. These inefficiently sequenced libraries may be amended by optimizing the primer sequences of the first and second PCR used to construct the ms4C-seq library. After the completion of our work, the UMI-4C method, which is similar to ms4C-seq, was reported ${ }^{50}$, supporting the general utility of these methods for examining chromatin interactions.

The subnuclear localization of gene loci affects gene expression. Inactive genes marked by transcriptionally silent histone modifications, such as $\mathrm{H} 3 \mathrm{~K} 27 \mathrm{me} 3$ and $\mathrm{H} 3 \mathrm{~K} 9 \mathrm{me} 3$, are enriched in the nuclear periphery, whereas transcriptionally active genes marked by H3K4me3 are localized in the nuclear interior ${ }^{41}, 51,52$. Furthermore, the artificial tethering of a gene to the nuclear membrane leads to repression of the active gene ${ }^{39}$, strongly supporting a causal relationship between nuclear positioning and gene expression. Our analyses showed that, along with the restoration of bivalency, gene loci at the nuclear periphery in HFs are located in the nuclear interior of hPSCs, and that the bivalent gene repositioning is accompanied by alterations in the nuclear position of the interaction target loci (Fig. 4; Supplementary Fig. 3). These results suggest that developmental gene loci might change their nuclear position from the nuclear periphery to the interior during somatic cell reprogramming, allowing the loci to be easily accessible to transcription factors and RNA polymerase in the nuclear interior of hPSCs.

In the nuclear interior, active genes colocalize in a distinct structure, termed the transcriptional factory, which may be important for the coregulation of gene expression ${ }^{44-46}$. In contrast, our results demonstrated that bivalent gene loci frequently colocalize in hPSCs (Fig. 5; Supplementary Fig. 6a), even though their expression was hardly observed. Similar to the concept of the transcriptional factory, the colocalization of bivalent gene loci may be crucial for the coregulation of developmental genes in hPSCs. By sharing regulatory factors, including transcription factors and RNA polymerase, the colocalization of bivalent gene loci in hPSCs may allow developmental genes to be coordinately transcribed as a rapid response to extracellular differentiation cues.

We also showed that histone modifiers contribute to the colocalization of bivalent genes in hPSCs. RING1B and EED, which are components of PRC1 and PRC2, respectively, are essential for the chromatin organization of polycomb-regulated gene loci in mouse PSCs ${ }^{53-55}$. Our results suggest that PRC1 and PRC2 are involved in broader aspects of chromatin interactions including bivalent gene colocalization. We also revealed that an active histone modifier, WDR5, is required for higher-order chromatin structures in hPSCs (Fig. 6; Supplementary Fig. 7c). Recent studies have indicated that noncoding RNAs are important for the construction of chromatin interactions ${ }^{56,57}$. Interestingly, several enhancer-like long noncoding RNAs (lncRNAs) also play a role in recruiting WDR5 to target loci for the methylation of $\mathrm{H} 3 \mathrm{~K} 4^{58-60}$. Therefore, in addition to histone modifiers, IncRNAs may also mediate the colocalization of bivalent genes.

Our knockdown experiments showed that the contribution of RING1B, EED, and WDR5 to the colocalization of bivalent genes varies among baits. These different effects on the colocalization of bivalent gene loci may have several causes, including different cofactors, binding strengths, frequencies, and stabilities of the histone modifiers. Knockout of RING1B and EED derepresses developmental genes in ESCs ${ }^{11,61}$, and WDR5 is required for ESC self-renewal ${ }^{62}$. Therefore, the colocalization of bivalent genes that are mediated by histone modifiers may be a critical regulatory event underlying the differentiation and self-renewal capabilities of PSCs.

In conclusion, our present study shows that bivalent gene loci colocalize to the nuclear interior of hPSCs, and that the organization of chromatin 3D structures around bivalent genes is mediated by histone modifiers related not only to inactive but also to active marks (Fig. 6e). Because bivalent histone modifications increase during the transition from the morula to the inner cell mass or trophectoderm in early embryogenesis ${ }^{63}$, the detailed regulatory mechanisms of higher-order chromatin structures and subnuclear localization at bivalent gene loci should provide insights on early embryogenesis.

\section{Methods}

Cell culture conditions. hESC lines (H9 and KhES3) and hiPSC lines (428C2 and 409B2) ${ }^{64,65}$ were cultured in DMEM/F12 (Thermo Fisher 10565-018) supplemented with $20 \%$ knockout serum replacement (Thermo Fisher 10828-028), 2 mM L-glutamine (Thermo Fisher 25030-081), 1× nonessential amino acids (Thermo Fisher 11140-050), 110 mM 2-mercaptoethanol (Thermo Fisher 21985-023), penicillin/streptomycin (0.05 U, $50 \mu \mathrm{g}$ per ml) (Thermo Fisher 15140-122), and 4 ng per ml of hbFGF (Wako 064-054541) on feeder layers of mitomycin-C-treated SNL-STO cells stably expressing the puromycin resistance gene. HFs (1388 and TIG120) were cultured in DMEM (Nacalai Tesque 08459-64) supplemented with 10\% FBS (Japan Bio Serum S1560-500). The mouse SNL-STO cells used as feeder cells were cultured in DMEM (Nacalai Tesque 08459-64) supplemented with $10 \%$ FBS (Thermo Fisher 10437-028), $0.05 \mathrm{U}$ per $\mathrm{ml}$ of penicillin, and $50 \mu \mathrm{g}$ per $\mathrm{ml}$ of penicillin/streptomycin ( $0.05 \mathrm{U}, 50 \mu \mathrm{g}$ per l) (Thermo Fisher 15140-122). All cells were cultured at $37^{\circ} \mathrm{C}$ in a $5 \% \mathrm{CO}_{2}$ incubator. KhES3 was obtained from Kyoto University. H9 was from WiCell Research Institute. TIG120 was obtained from the Japanese Collection of Research Bioresources. HDF 1388 was purchased from Cell Applications.

Vector construction. shRNA expression vectors were constructed by cloning the hU6 promoter and puromycin resistance genes between piggyBac-inverted terminal repeats ${ }^{66}$. Target sequences of shRNAs for EED, RING1B, and WDR5 were designed using siDirect (http://sidirect2.rnai.jp/) and cloned downstream of the hU6 promoter of the modified vector (Supplementary Fig. 7a; Supplementary Table 1). The CAG promoter-driven expression vector of piggyBac transposase ${ }^{67}$ was cotransfected with the shRNA expression vector.

Knockdown experiments. $428 \mathrm{C} 2$ cells were washed with $1 \times$ PBS, and feeder cells were removed by $3-5 \mathrm{~min}$ of incubation in dissociation solution (Repro Cell RCHETP002). The hiPSC colonies were dissociated into single cells by incubation in ACCUMAX (Innova Cell Technologies AM105) at $37^{\circ} \mathrm{C}$ for $10 \mathrm{~min}$. To prepare the transfection mix, $6 \mu \mathrm{g}$ of shRNA expression vector and $6 \mu \mathrm{g}$ of CAG promoterdriven expression vector of piggyBac transposase were mixed with $540 \mu \mathrm{l}$ of OptiMEM (Thermo Fisher 31985-062) and $46 \mu$ l of FuGENE HD (Promega E2311). After incubation of the transfection mix at RT for $15 \mathrm{~min}, 5 \times 10^{6}$ cells were resuspended in the transfection mix and incubated at RT for 5 min. shRNAtransfected cells were suspended in $6 \mathrm{ml}$ of mTeSR1 medium (Stemcell Technologies 05850) in the presence of $10 \mu \mathrm{M}$ Y-27632 (Wako 257-00511) on Matrigel (CORNING 356231)-coated 10-cm dishes. Selection with $2 \mu \mathrm{g}$ per ml puromycin was performed 2 days after transfection. The cells were harvested 4 days after transfection for ms4C-seq analysis, immunoblotting, and quantitative PCR (qPCR) analysis.

RNA isolation, cDNA synthesis, and qPCR. RNA was extracted from each cell line with RNeasy Mini Kit (QIAGEN 74104). Complementary DNA was synthesized from $100 \mathrm{ng}$ of total RNAs with QuantiTect Reverse Transcription Kit (Qiagen). qPCR analysis was performed with SYBR premix Ex TaqII (Takara) and primer sets (Supplementary Table 1) on StepOnePlus Real-Time PCR System (Applied Biosystems). Expression levels were normalized to that of GAPDH.

Microarray experiments. RNA was extracted from hiPSCs (409B2) and the original HFs (1388) with TRIzol and QIAGEN RNeasy Kit. The total RNA was subjected to cRNA synthesis with $3^{\prime}$ IVT Express Kit (Affymetrix), and the resultant cRNA was fragmented and hybridized to the HG-U133 Plus 2 platform (Affymetrix). After hybridization, GeneChip arrays were washed, stained with 
GeneChip Fluidics Station 450 (Affymetrix), and detected with Scanner 3000 TG System (Affymetrix) following the manufacturer's standard protocols. Data analyses were performed with GeneSpringGX 13.0 software (Agilent Technologies).

Immunoblotting. Cell lysates were prepared from an equal number of cells $\left(0.5-1 \times 10^{6}\right.$ cells), which were suspended in $100 \mu \mathrm{l}$ of CytoBuster Protein Extraction Reagent (BIO RAD 71009-50ML) supplemented with $1 \times$ protease inhibitor cocktail (Roche 04693159 001) followed by the addition of $100 \mu \mathrm{l}$ of Laemmli sample buffer (Bio-rad 161-0737) in the presence of 5\% 2mercaptoethanol (Nacalai 21438-82). SDS-PAGE was performed with $10 \%$ gels (Bio-rad 456-1036) or gradient gels (Bio-rad 456-9036) in 1× Tris/Glycine/SDS buffer (Bio-rad 161-0732). The separated proteins were transferred to a PVDF membrane (Bio-rad 162-0174). Target proteins were detected with the following antibodies: anti-EED (1:1000; rabbit; Millipore 09-774), anti-WDR5 (1:1000; rabbit; Millipore 07-706), anti-RING1B (1:500; rabbit; Cell Signaling Technology D22F2), and anti-GAPDH (1:4000; Ambion AM4300). Secondary antibodies were AntiRabbit IgG, HRP-Linked F(ab')2 Fragment Donkey (1:4000; NA9340; GE Healthcare Life Science), or Anti-Mouse IgG, HRP-Linked F( $\left.a b^{\prime}\right) 2$ Fragment Sheep (1:4000; NA9310; GE Healthcare Life Science).

3D DNA FISH. Probes were prepared from BAC clones (BACPAC Resources) by using a Nick Translation Reagent Kit (Abbott Molecular Inc. 32-801300) and labeled with Red 650 dUTP (ENZO ENZ-42522), Red 580 dUTP (ENZO ENZ42844), or Green 496 dUTP (ENZO ENZ-42837). Nick-translated probes were precipitated with $70 \% \mathrm{EtOH}(1 \mu \mathrm{g} / \mu \mathrm{l}$ human CotI DNA, $10 \mu \mathrm{g}$ per $\mu \mathrm{l}$ of salmon sperm DNA, and $3 \mathrm{M}$ sodium acetate). The probes were dissolved in $4 \mu \mathrm{l}$ of formamide that was preheated at $42^{\circ} \mathrm{C}$, denatured at $75^{\circ} \mathrm{C}$ for $10 \mathrm{~min}$, and then placed on ice for $3 \mathrm{~min}$. The denatured probes were dissolved in $1 \times$ hybridization buffer ( $2 \mathrm{mg}$ per $\mathrm{ml}$ of BSA (Roche 711454 ), $2 \times$ SSC, and $10 \%$ dextran sulfate sodium salt (Sigma D8906)). Cells cultured on gelatin-coated chamber slides (Iwaki) were fixed with 4\% PFA in PBS at room temperature for $10 \mathrm{~min}$ and permeabilized with $0.5 \%$ Triton X-100 in PBS at RT for 5-15 min. Permeabilized cells were washed two times with $70 \% \mathrm{EtOH}$ that was prechilled at $-20^{\circ} \mathrm{C}$ and dehydrated in 70, 90, and $100 \% \mathrm{EtOH}$ ( $\mathrm{v}$ per v) at RT for 5 min each. DNA in the nuclei was denatured in buffer $(70 \%$ formamide $(\mathrm{pH} 7.0), 2 \times \mathrm{SSC})$ at $80^{\circ} \mathrm{C}$ for 5 $\mathrm{min}$. The denatured samples were washed and dehydrated with a gradient of icecold ethanol (70, 90, and 100\% EtOH (v per v)) for 5 min each. The dissolved probes were hybridized to the samples in a $37^{\circ} \mathrm{C}$ wet box for $1-2$ days. The samples were then washed three times with $50 \%$ formamide $/ 2 \times \mathrm{SSC}$ solution at $42{ }^{\circ} \mathrm{C}$ for 10 min and then washed two more times with $2 \times$ SSC solution at $42^{\circ} \mathrm{C}$ for $5 \mathrm{~min}$. The nuclei were counterstained with $2 \mu \mathrm{g}$ per $\mathrm{ml}$ of Hoechst at RT for $10 \mathrm{~min}$. FISH signals were detected by using Zeiss LSM710. Z sections that were captured at 0.2$\mu \mathrm{m}$ intervals. The following BACs were used in these studies: RP11-235I5 (GATA4), RP11-102O8 (LGI3), RP11-383B10 (MTUS1), RP11-1083M23 (TWIST1), RP11-815K24 (EGFR), and RP11-614D15 (INHBA). Distances between the bait probe and the positive or negative probes were measured in 180-456 signal pairs from three to five visual fields in a single experiment and the number of colocalized signals was counted. The statistical significance was calculated by Fisher's exact test.

3C library preparation and 3C-qPCR assay. The 3C assays in this study were performed according to the following protocol ${ }^{22}$. Briefly, $1-2 \times 10^{7}$ cells were crosslinked in $9.25 \mathrm{ml}$ of ES cell culture medium containing $1 \%$ formaldehyde (Wako 064-00406) at RT for $10 \mathrm{~min}$. The formaldehyde was quenched with $500 \mu \mathrm{l}$ of $2.5 \mathrm{M}$ glycine and washed twice with $5 \mathrm{ml}$ of ice-cold $1 \times$ PBS. Cross-linked nuclei were isolated by incubation in $550 \mu \mathrm{l}$ of lysis buffer $(10 \mathrm{mM}$ Tris- $\mathrm{HCl}, \mathrm{pH} 7.5,10 \mathrm{mM}$ $\mathrm{NaCl}, 0.2 \% \mathrm{~Np}-40$, and $1 \times$ protease inhibitor (Roche 04693159001 )) on ice for 20 min and homogenization with 10 strokes $\times 2$ by a tight pestle in a dounce homogenizer (Wheaton) on ice. The isolated nuclei were washed twice with $500 \mu \mathrm{l}$ of $1 \times$ cutsmart buffer (NEB B7200S). The formaldehyde was quenched with $500 \mu \mathrm{l}$ of $2.5 \mathrm{M}$ glycine and washed twice with $5 \mathrm{ml}$ of ice-cold $1 \times \mathrm{PBS}$. Cross-linked nuclei were isolated by incubation in $550 \mu$ of lysis buffer $(10 \mathrm{mM}$ Tris- $\mathrm{HCl}, \mathrm{pH}$ 7.5, $10 \mathrm{mM} \mathrm{NaCl}, 0.2 \% \mathrm{~Np}-40$, and $1 \times$ protease inhibitor (Roche 04693159001 )) on ice for $20 \mathrm{~min}$ and homogenization with 10 strokes $\times 2$ by a tight pestle in a dounce homogenizer (Wheaton) on ice. The isolated nuclei were washed twice with $500 \mu \mathrm{l}$ of $1 \times$ cutsmart buffer (NEB B7200S). Proximity ligation of the HindIIIdigested DNA ends was conducted with $10 \mathrm{U}$ (Weiss unit) of T4 DNA ligase (TAKARA 2011A). 3C DNA libraries were purified by phenol-chloroform extractions (Nacarai 25970-56, 26829-96) and ethanol precipitation. Precipitated $3 \mathrm{C}$ libraries were purified again by using an Invitrogen PureLink Genomic DNA Mini Kit (Thermo Fisher K1820-00). The 3C-qPCR experiments were performed by using a SYBR premix Ex TaqII (Takara) and primer sets (Supplementary Table 1) on a StepOnePlus Real-Time PCR System (Applied Biosystems). For qPCR, $100 \mathrm{ng}$ of template was used for each reaction, and the reaction steps were 1 cycle at $95^{\circ} \mathrm{C}$ for $30 \mathrm{~s}$ and 45 cycles at $95^{\circ} \mathrm{C}$ for $5 \mathrm{~s}$, and 1 cycle at $60^{\circ} \mathrm{C}$ for $30 \mathrm{~s}$. Interaction frequencies were normalized to the DNA amount based on measurements using the GAPDH locus.
ms4C-seq and library construction for enhanced 3C-qPCR. The 3C libraries were sonicated into 300-700-bp-long fragments in microtubes (Covaris 520045) with Covaris E210 under the following conditions: duty cycle 5\%, intensity 3, cycles per burst 200, and time $150 \mathrm{~s}$. End-repairing and A-tailing were performed by using NEBNext Ultra End Repair/dA-Tailing Module (NEB E7442S) according to the manufacturer's instructions. Ligation of the Splinkerette adaptor ${ }^{23}$ for sequencing by the next-generation sequencer (Top: 5'-CGAAGAGTAACCGTTGCTAGGAGAGACCGTGACTGGAGTTCAGACGTGTGCTCTTCCGATCT-3', Bottom: 3'phosphate-GATCGGAAGAGCTGTTTTTTTTTTCAAAAAAA-5') to the A-tailed $3 \mathrm{C}$ libraries was performed with Ligation high Ver.2 (TOYOBO LGK-201) at $16^{\circ} \mathrm{C}$ for $30 \mathrm{~min}$ and 10:1 adapter:library molar ratio. The adaptor-ligated $3 \mathrm{C}$ libraries were purified with $1.8 \times$ AMPureXP beads. A total of $3.7 \mu \mathrm{g}(230 \mathrm{ng} /$ reaction $\times 16)$ of adaptor-ligated 3C libraries served as the template to amplify target loci by PCR amplification. Each PCR was performed in a 50- $\mu$ l reaction mix (1× Phusion HighFidelity PCR Master Mix with HF Buffer (NEB M0531S), $50 \mathrm{nM}$ each universal and target primers (Supplementary Table 1), and $230 \mathrm{ng} /$ tube template). The reaction steps were 1 cycle at $98^{\circ} \mathrm{C}$ for $30 \mathrm{~s}, 20$ cycles at $98^{\circ} \mathrm{C}$ for $10 \mathrm{~s}, 62^{\circ} \mathrm{C}$ for $30 \mathrm{~s}$, and 72 ${ }^{\circ} \mathrm{C}$ for $30 \mathrm{~s}$; and $1 \mathrm{cycle}$ at $72{ }^{\circ} \mathrm{C}$ for $10 \mathrm{~min}$. Samples after the first PCR amplification were also used for 3C-qPCR assays as templates of enhanced 3C-qPCR (Fig. 3d). To reduce the influence of different amplification efficiencies among primer sets, a second nested PCR was performed in the 13 bait groups, and it had similar amplification efficiencies (data not shown). The reaction steps for the second PCR were 1 cycle at $98^{\circ} \mathrm{C}$ for $30 \mathrm{~s}, 20$ cycles at $98^{\circ} \mathrm{C}$ for $10 \mathrm{~s}, 60^{\circ} \mathrm{C}$ for $30 \mathrm{~s}$, and $72{ }^{\circ} \mathrm{C}$ for $30 \mathrm{~s}$; and $1 \mathrm{cycle}$ at $72^{\circ} \mathrm{C}$ for $10 \mathrm{~min}$ (second universal and target primers (Supplementary Table 1). Amplified multiplexed splinkerette 3C libraries were electrophoresed on a $2 \%$ agarose gel (Bio-Rad 1613107), and libraries between 350 and 700 bp were excised and purified with QIAquick Gel Extraction Kit (QIAGEN 28704). This library was quantified with Library Quantification Kit (KAPA KK4824) and sequenced via HiSeq 2000 sequencing with paired-end reads of $100 \mathrm{bp}$ from both ends or NextSeq 500 with paired-end reads of $80 \mathrm{bp}$ from both ends. For the POU5F1 locus, we were unable to design a specific second primer (second universal and target primers (Supplementary Table 1)), because its sequence is present in three loci: chr. 6, chr. 12, and chr. 1. However, we used this second primer to amplify the POU5F1 locus, because the first PCR primer for POU5F1 is specific to the bait locus.

Analysis of ms4C-seq data. ms4C-seq data sets were analyzed according to the following procedures. Bases with low-quality scores and the adapters in all sequenced reads were trimmed with Cutadapt-1.4.2 ${ }^{68}$. Read pairs with the HindIII site "AAGCTT" in the read1 (R1) sequence were used for the following analysis. The R1 sequences were separated into bait sequences and target sequences. To divide the reads into each bait, the former parts from HindIII of R1 were excised as bait sequence and mapped to 47 bait sequences by BWA (bwa-0.6.2) ${ }^{69}$ by using the default setting. The NANOG pseudogene gene loci were discriminated from NANOG bait loci on the basis of an SNP in the NANOG region. The latter parts from HindIII of the R1 and R2 read pairs were used for the subsequent mapping These read pair sequences were mapped to the human reference genome (hg19) by BWA (bwa-0.6.2). The read pairs, which were derived from self-ligation and nodigestion events, were removed. PCR duplicates were removed by Picard (Picardtools-1.97). The total read numbers within $1000 \mathrm{bp}$ from each HindIII site were counted, and the counted numbers in the HindIII sites were collected as wiggle (.wig) format files. The total read numbers and the ratio of cis:trans reads were used for quality control (Supplementary Table 2). To exclude the baits, which have insufficient sequenced reads for further analysis, percentages of the observed cis interactions in the expected total number of $c i s$ interactions were calculated for each bait. The total number of distinct $c i s$ interactions was estimated by using Preseq software ${ }^{70}$.

Domainograms and calculation of interaction frequencies. Using the read count data at each HindIII site (wiggle format data), we analyzed the contact probabilities of the HindIII site by the following method ${ }^{33}$. In our analysis, instead of running windows for the number of HindIII sites, running windows for distances from each HindIII site were used to obtain contact probabilities. The enrichment level of reads in small windows (within $100 \mathrm{~Kb}$ from the HindIII) for reads in large windows (within $3 \mathrm{Mb}$ from the HindIII) was statistically calculated as contact probabilities in each HindIII. Two statistical methods, the $U$-test and the binomial test, were used to calculate contact probabilities in proximal regions (within 8-Mb regions) and the distal region (distance $>8 \mathrm{Mb}$ ) from the baits, respectively. The contact probabilities in each HindIII site are presented in domainograms by using previously published programs $s^{37}$. Gene-to-gene contact probabilities were calculated around the TSS of each gene through the same method described above with the one-sided binomial test, and adjusted by FDR.

Correlation analysis of chromatin interactions. To compare chromatin interaction profiles between biological duplicates or cell types, the following analysis was performed ${ }^{53}$. Briefly, wiggle-formatted ms4C-seq data in each HindIII were binarized to 1 (reads $>0)$ and $0($ read $=0)$, and cis-hit coverages were calculated by averaging these binarized values in a sliding window (100 kb from each HindIII 
site). Spearman correlation coefficients were calculated by comparing the cis-hit coverages between cell types or biological duplicates.

Definition of transcriptional gene states. Chromatin states data of hESCs and NHLFs were obtained from the ENCODE project data sets of UCSC (http:// hgdownload.cse.ucsc.edu/goldenpath/hg19/encodeDCC/wgEncodeBroadHmm/ wgEncodeBroadHmmH1hescHMM.CNV.bed, http://hgdownload.cse.ucsc.edu/ goldenpath/hg19/encodeDCC/wgEncodeBroadHmm/wgEncodeBroadHmmNhlf HMM.bed.gz ${ }^{29}$ and used for the gene definitions. We defined the refseq genes that are mainly occupied by "poised promoter", "Active/Weak Promoter", and "Repressed/Heterochrom" chromatin states as "bivalent genes", "active genes", and "repressive genes", respectively.

Analysis of LADs. For nuclear localization analysis of gene loci, we used publically available $\log _{2}$ (Dam-LMNB1/Dam) signal data of Tig3 $\mathrm{HFs}^{41}$ (GSE8854) as HFs and of SHEF-2 human ESCs ${ }^{40}$ (GSE22428) as PSCs, and define the laminaassociated domains (LADs) according to a previously reported method ${ }^{41}$. Briefly, for the identification of LADs, the $\log _{2}$ (Dam-LMNB1/Dam) signal was binarized with positive values to 1 and negative values to -1 . Transition sites of these signals were identified as left and right side edges of LADs by the sliding filter, which compared the average binary values between the right and left 99-probe windows of the probe ${ }^{40,41}$. Cumulative fraction analysis for $\log _{2}(\mathrm{Dam}-\mathrm{LMNB} / \mathrm{Dam})$ signals in each gene was performed using the average $\log _{2}($ Dam-LMNB1/Dam) values for each probe in the gene bodies.

Data availability. All DNA microarray data are deposited in Gene Expression Omnibus under the accession number for GSE90141.

All ms4C-seq data are deposited in Gene Expression Omnibus under the accession number for GSE90782.

Received: 25 January 2017 Accepted: 6 October 2017

Published online: 20 November 2017

\section{References}

1. Bernstein, B. E. et al. A bivalent chromatin structure marks key developmental genes in embryonic stem cells. Cell 125, 315-326 (2006).

2. $\mathrm{Ku}, \mathrm{M}$. et al. Genomewide analysis of PRC1 and PRC2 occupancy identifies two classes of bivalent domains. PLoS Genet. 4, e1000242 (2008).

3. Pan, G. et al. Whole-genome analysis of histone H3 lysine 4 and lysine 27 methylation in human embryonic stem cells. Cell Stem Cell 1, 299-312 (2007).

4. Zhao, X. D. et al. Whole-genome mapping of histone H3 Lys4 and 27 trimethylations reveals distinct genomic compartments in human embryonic stem cells. Cell Stem Cell 1, 286-298 (2007).

5. Azuara, V. et al. Chromatin signatures of pluripotent cell lines. Nat. Cell Biol. 8 , 532-538 (2006).

6. Dou, Y. et al. Physical association and coordinate function of the $\mathrm{H} 3 \mathrm{~K} 4$ methyltransferase MLL1 and the H4 K16 acetyltransferase MOF. Cell 121, 873-885 (2005).

7. Kuzmichev, A., Nishioka, K., Erdjument-Bromage, H., Tempst, P. \& Reinberg, D. Histone methyltransferase activity associated with a human multiprotein complex containing the Enhancer of Zeste protein. Genes Dev. 16, 2893-2905 (2002).

8. Wysocka, J. et al. WDR5 associates with histone $\mathrm{H} 3$ methylated at $\mathrm{K} 4$ and is essential for H3 K4 methylation and vertebrate development. Cell 121, 859-872 (2005).

9. Brookes, E. et al. Polycomb associates genome-wide with a specific RNA polymerase II variant, and regulates metabolic genes in ESCs. Cell Stem Cell 10, 157-170 (2012)

10. Min, I. M. et al. Regulating RNA polymerase pausing and transcription elongation in embryonic stem cells. Genes Dev. 25, 742-754 (2011).

11. Stock, J. K. et al. Ring1-mediated ubiquitination of H2A restrains poised RNA polymerase II at bivalent genes in mouse ES cells. Nat. Cell Biol. 9, 1428-1435 (2007).

12. Polo Jose, M. et al. A molecular roadmap of reprogramming somatic cells into iPS Cells. Cell 151, 1617-1632 (2012).

13. Morin-Kensicki, E. M., Faust, C., LaMantia, C. \& Magnuson, T. Cell and tissue requirements for the gene eed during mouse gastrulation and organogenesis. Genesis 31, 142-146 (2001).

14. Pasini, D., Bracken, A. P., Jensen, M. R., Lazzerini Denchi, E. \& Helin, K. Suz12 is essential for mouse development and for EZH2 histone methyltransferase activity. EMBO J. 23, 4061-4071 (2004).

15. O'Carroll, D. et al. The polycomb-group gene Ezh2 is required for early mouse development. Mol. Cell Biol. 21, 4330-4336 (2001).
16. Jost, K. L. et al. 3D-image analysis platform monitoring relocation of pluripotency genes during reprogramming. Nucleic Acids Res. 39, e113 (2011).

17. Apostolou, E. et al. Genome-wide chromatin interactions of the Nanog locus in pluripotency, differentiation, and reprogramming. Cell Stem Cell 12, 699-712 (2013).

18. de Wit, E. \& de Laat, W. A decade of 3 C technologies: insights into nuclear organization. Genes Dev. 26, 11-24 (2012).

19. Gheldof, N., Leleu, M., Noordermeer, D., Rougemont, J. \& Reymond, A. Detecting long-range chromatin interactions using the chromosome conformation capture sequencing (4C-seq) method. Methods Mol. Biol. 786, 211-225 (2012).

20. Simonis, M. et al. Nuclear organization of active and inactive chromatin domains uncovered by chromosome conformation capture-on-chip (4C). Nat. Genet. 38, 1348-1354 (2006).

21. Zhao, Z. et al. Circular chromosome conformation capture (4C) uncovers extensive networks of epigenetically regulated intra- and interchromosomal interactions. Nat. Genet. 38, 1341-1347 (2006).

22. Lieberman-Aiden, E. et al. Comprehensive mapping of long-range interactions reveals folding principles of the human genome. Science 326, 289-293 (2009).

23. Devon, R. S., Porteous, D. J. \& Brookes, A. J. Splinkerettes--improved vectorettes for greater efficiency in PCR walking. Nucleic Acids Res. 23, 1644-1645 (1995).

24. Huang, P. Y. H. et al. Protocol: sonication-based circular chromosome conformation capture with next-generation sequencing analysis for the detection of chromatin interactions. Protoc. Exch., doi: https://doi.org/10.1038/ protex.2010.1207 (2010).

25. Stadhouders, R. et al. Multiplexed chromosome conformation capture sequencing for rapid genome-scale high-resolution detection of long-range chromatin interactions. Nat. Protoc. 8, 509-524 (2013).

26. Wei, Z. et al. Klf4 organizes long-range chromosomal interactions with the oct 4 locus in reprogramming and pluripotency. Cell Stem Cell 13, 36-47 (2013).

27. Ernst, J. \& Kellis, M. ChromHMM: automating chromatin-state discovery and characterization. Nat. Methods 9, 215-216 (2012).

28. Ernst, J. et al. Mapping and analysis of chromatin state dynamics in nine human cell types. Nature 473, 43-49 (2011).

29. ENCODE Project Consortium. An integrated encyclopedia of DNA elements in the human genome. Nature 489, 57-74 (2012).

30. Mikkelsen, T. S. et al. Genome-wide maps of chromatin state in pluripotent and lineage-committed cells. Nature 448, 553-560 (2007).

31. Voigt, P., Tee, W. W. \& Reinberg, D. A double take on bivalent promoters Genes Dev. 27, 1318-1338 (2013).

32. Drukker, M. et al. Isolation of primitive endoderm, mesoderm, vascular endothelial and trophoblast progenitors from human pluripotent stem cells. Nat. Biotechnol. 30, 531-542 (2012).

33. Albini, S. et al. Epigenetic reprogramming of human embryonic stem cells into skeletal muscle cells and generation of contractile myospheres. Cell Rep. 3, 661-670 (2013).

34. Yu, P., Pan, G., Yu, J. \& Thomson, J. A. FGF2 sustains NANOG and switches the outcome of BMP4-induced human embryonic stem cell differentiation. Cell Stem Cell 8, 326-334 (2011).

35. Vodyanik, M. A. et al. A mesoderm-derived precursor for mesenchymal stem and endothelial cells. Cell Stem Cell 7, 718-729 (2010).

36. de Wit, E., Braunschweig, U., Greil, F., Bussemaker, H. J. \& van Steensel, B. Global chromatin domain organization of the Drosophila genome. PLoS Genet. 4, e1000045 (2008).

37. Splinter, E., de Wit, E., van de Werken, H. J., Klous, P. \& de Laat, W. Determining long-range chromatin interactions for selected genomic sites using 4C-seq technology: from fixation to computation. Methods 58, 221-230 (2012).

38. Peric-Hupkes, D. et al. Molecular maps of the reorganization of genomenuclear lamina interactions during differentiation. Mol. Cell 38, 603-613 (2010).

39. Finlan, L. E. et al. Recruitment to the nuclear periphery can alter expression of genes in human cells. PLoS Genet. 4, e1000039 (2008).

40. Meuleman, W. et al. Constitutive nuclear lamina-genome interactions are highly conserved and associated with A/T-rich sequence. Genome Res. 23, 270-280 (2013).

41. Guelen, L. et al. Domain organization of human chromosomes revealed by mapping of nuclear lamina interactions. Nature 453, 948-951 (2008).

42. Reddy, K. L., Zullo, J. M., Bertolino, E. \& Singh, H. Transcriptional repression mediated by repositioning of genes to the nuclear lamina. Nature 452, 243-247 (2008).

43. Luo, L. et al. The nuclear periphery of embryonic stem cells is a transcriptionally permissive and repressive compartment. J. Cell Sci. 122, 3729-3737 (2009).

44. Fanucchi, S., Shibayama, Y., Burd, S., Weinberg, M. S. \& Mhlanga, M. M. Chromosomal contact permits transcription between coregulated genes. Cell 155, 606-620 (2013). 
45. Iborra, F. J., Pombo, A., Jackson, D. A. \& Cook, P. R. Active RNA polymerases are localized within discrete transcription "factories' in human nuclei. J. Cell Sci. 109, 1427-1436 (1996).

46. Jackson, D. A., Hassan, A. B., Errington, R. J. \& Cook, P. R. Visualization of focal sites of transcription within human nuclei. EMBO J. 12, 1059-1065 (1993).

47. Guenther, M. G., Levine, S. S., Boyer, L. A., Jaenisch, R. \& Young, R. A. A chromatin landmark and transcription initiation at most promoters in human cells. Cell 130, 77-88 (2007).

48. Xie, W. et al. Epigenomic analysis of multilineage differentiation of human embryonic stem cells. Cell 153, 1134-1148 (2013).

49. Nagano, T. et al. Comparison of Hi-C results using in-solution versus innucleus ligation. Genome Biol. 16, 175 (2015).

50. Schwartzman, O. et al. UMI-4C for quantitative and targeted chromosomal contact profiling. Nat. Methods 13, 685-691 (2016).

51. Fu, Y. et al. MacroH2A1 associates with nuclear lamina and maintains chromatin architecture in mouse liver cells. Sci. Rep. 5, 17186 (2015).

52. Sadaie, M. et al. Redistribution of the Lamin B1 genomic binding profile affects rearrangement of heterochromatic domains and SAHF formation during senescence. Genes Dev. 27, 1800-1808 (2013).

53. Denholtz, M. et al. Long-range chromatin contacts in embryonic stem cells reveal a role for pluripotency factors and polycomb proteins in genome organization. Cell Stem Cell 13, 602-616 (2013).

54. Joshi, O. et al. Dynamic reorganization of extremely long-range promoterpromoter interactions between two states of pluripotency. Cell Stem Cell 17, 748-757 (2015)

55. Schoenfelder, S. et al. Polycomb repressive complex PRC1 spatially constrains the mouse embryonic stem cell genome. Nat. Genet. 47, 1179-1186 (2015).

56. Li, W. et al. Functional roles of enhancer RNAs for oestrogen-dependent transcriptional activation. Nature 498, 516-520 (2013).

57. Lai, F. et al. Activating RNAs associate with mediator to enhance chromatin architecture and transcription. Nature 494, 497-501 (2013).

58. Wang, K. C. et al. A long noncoding RNA maintains active chromatin to coordinate homeotic gene expression. Nature 472, 120-124 (2011)

59. Gomez, J. A. et al. The NeST long ncRNA controls microbial susceptibility and epigenetic activation of the interferon-gamma locus. Cell 152, 743-754 (2013).

60. Yang, Y. W. et al. Essential role of lncRNA binding for WDR5 maintenance of active chromatin and embryonic stem cell pluripotency. Elife 3, e02046 (2014).

61. Boyer, L. A. et al. Polycomb complexes repress developmental regulators in murine embryonic stem cells. Nature 441, 349-353 (2006).

62. Ang, Y. S. et al. Wdr5 mediates self-renewal and reprogramming via the embryonic stem cell core transcriptional network. Cell 145, 183-197 (2011).

63. Liu, X. et al. Distinct features of H3K4me3 and H3K27me3 chromatin domains in pre-implantation embryos. Nature 537, 558-562 (2016).

64. Nishizawa, M. et al. Epigenetic variation between human induced pluripotent stem cell lines is an indicator of differentiation capacity. Cell Stem Cell 19, 341-354 (2016).

65. Okita, K. et al. A more efficient method to generate integration-free human iPS cells. Nat. Methods 8, 409-412 (2011).

66. Woltjen, K. et al. piggyBac transposition reprograms fibroblasts to induced pluripotent stem cells. Nature 458, 766-770 (2009).

67. Yoshida, Y., Takahashi, K., Okita, K., Ichisaka, T. \& Yamanaka, S. Hypoxia enhances the generation of induced pluripotent stem cells. Cell Stem Cell 5, 237-241 (2009)

68. Martin, M. Cutadapt removes adapter sequences from high-throughput sequencing reads. EMBnet J. 17, 10-12 (2011).
69. Li, H. \& Durbin, R. Fast and accurate short read alignment with BurrowsWheeler transform. Bioinformatics 25, 1754-1760 (2009).

70. Daley, T. \& Smith, A. D. Predicting the molecular complexity of sequencing libraries. Nat. Methods 10, 325-327 (2013)

\section{Acknowledgements}

We thank the members of our laboratory for their valuable scientific discussions and administrative support. We thank M. Kabata, T. Sato and S. Sakurai for their technical assistance; K. Okita for the hiPSCs, hESCs, and plasmid; S. Ohta for shRNA design; I. Okamoto and M. Saitou for instruction on the 3D DNA FISH method; and J. Lee, P. Gee, K. Takahashi, S. Masui, H. Saito and P. Karagiannis for reviewing the manuscript. This work was supported in part by AMED-CREST, AMED; Core Center for iPS Cell Research, Research Center Network for Realization of Regenerative Medicine from Japan Agency for Medical Research and Development, AMED; JSPS KAKENHI Grant Number 23790329 and 15H01352; iPS Cell Research Fund; and the Grant-in-Aid for JSPS DC2 research fellowship 14J01800. S.Y. is a scientific advisor of iPS Academia Japan without salary. The iCeMS is supported by World Premier International Research Center Initiative (WPI), MEXT, Japan.

\section{Author contributions}

Conceptualization: H.I., M.S. and T.Y.; Methodology: H.I., M.S. and T.Y.; Software: H.I and T.Y.; Validation: H.I.; Formal analysis: H.I. and T.Y.; Investigation: H.I.; Writing original draft: H.I. and T.Y.; Writing, review, and editing: H.I., M.S. and T.Y.; Resources: H.I., M.S., S.Y. and T.Y.; Funding acquisition: H.I., M.S., S.Y. and T.Y.; and Supervision: S.Y. and T.Y.

\section{Additional information}

Supplementary Information accompanies this paper at doi:10.1038/s41467-017-01679-x

Competing interests: The authors declare no competing financial interests.

Reprints and permission information is available online at http://npg.nature.com/ reprintsandpermissions/

Publisher's note: Springer Nature remains neutral with regard to jurisdictional claims in published maps and institutional affiliations.

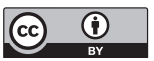

Open Access This article is licensed under a Creative Commons Attribution 4.0 International License, which permits use, sharing, adaptation, distribution and reproduction in any medium or format, as long as you give appropriate credit to the original author(s) and the source, provide a link to the Creative Commons license, and indicate if changes were made. The images or other third party material in this article are included in the article's Creative Commons license, unless indicated otherwise in a credit line to the material. If material is not included in the article's Creative Commons license and your intended use is not permitted by statutory regulation or exceeds the permitted use, you will need to obtain permission directly from the copyright holder. To view a copy of this license, visit http://creativecommons.org/ licenses/by/4.0/.

(c) The Author(s) 2017 